\title{
Interplay between polarization, strain, and defect pairs in Fe-doped $\mathrm{SrMnO}_{3-\delta}$
}

\author{
Chiara Ricca and Ulrich Aschauer $\odot^{*}$ \\ Department of Chemistry and Biochemistry, University of Bern, Freiestrasse 3, CH-3012 Bern, Switzerland \\ and National Centre for Computational Design and Discovery of Novel Materials (MARVEL), CH-1015 Lausanne, Switzerland
}

(Received 15 June 2021; revised 21 July 2021; accepted 20 August 2021; published 10 September 2021)

\begin{abstract}
Defect chemistry, strain, and structural, magnetic, and electronic degrees of freedom constitute a rich space for the design of functional properties in transition-metal oxides. Here, we show that it is possible to engineer polarity and ferroelectricity in nonpolar perovskite oxides via polar defect pairs formed by anion vacancies coupled to substitutional cations. We use a self-consistent site-dependent approach employing with a correction that accounts for local structural and chemical changes upon defect creation and which is crucial to reconcile predictions with the available experimental data. Our results for Fe-doped oxygen-deficient $\mathrm{SrMnO}_{3}$ show that substitutional $\mathrm{Fe}$ and oxygen vacancies can promote polarity due to an off-center displacement of the defect charge resulting in a net electric dipole moment, which polarizes the lattice in the defect neighborhood. The formation of these defects and the resulting polarization can be tuned by epitaxial strain, resulting in enhanced polarization also for strain values lower than the ones necessary to induce a polar phase transition in undoped $\mathrm{SrMnO}_{3}$. For high enough defect concentrations, these defect dipoles couple in a parallel fashion, thus enabling defect- and strain-based engineering of ferroelectricity in $\mathrm{SrMnO}_{3}$.
\end{abstract}

DOI: 10.1103/PhysRevResearch.3.033237

\section{INTRODUCTION}

The interplay between electric polarization, magnetism, strain, and the defect chemistry constitutes a rich phase diagram for the design and control of novel functional properties in transition-metal perovskites [1-11]. In particular, strain imposed, for example, by lattice matching with the substrate during coherent epitaxial growth of thin films is an established route to engineer polarity and ferroelectricity in nonpolar complex oxides [5,7-10,12]. Defect engineering can tailor the ferroelectric response by introducing polar defect pairs. In particular, substitutional defects coupled to oxygen vacancies $\left(\mathrm{V}_{\mathrm{O}}\right)$, such as $\mathrm{Fe}_{\mathrm{Ti}}-\mathrm{V}_{\mathrm{O}}$ defect pairs, were shown to align with the direction of the lattice polarization in ferroelectric $\mathrm{PbTiO}_{3}$ [5] or to promote ferroelectricity in paraelectric $\mathrm{SrTiO}_{3}$ [13]. Polar distortions, strain, and stoichiometry can couple or compete in determining the material properties as shown for oxygen-deficient $\mathrm{SrMnO}_{3}$ (SMO) thin films [9], the material we also use as a model system in the present study.

Bulk SMO occurs in a hexagonal structure [14], but the perovskite phase of SMO (space group Pnma; see Fig. 1) with G-type antiferromagnetic (AFM) order [15] can be stabilized at low temperature in thin films [16]. It was predicted from theory that biaxial epitaxial strain induces a polar distortion in SMO, mainly associated with Mn ions displacing from their

\footnotetext{
*ulrich.aschauer@dcb.unibe.ch

Published by the American Physical Society under the terms of the Creative Commons Attribution 4.0 International license. Further distribution of this work must maintain attribution to the author(s) and the published article's title, journal citation, and DOI.
}

high-symmetry positions, the magnitude of the distortion and hence the ferroelectric polarization increasing with increasing biaxial strain $[8,9,12]$. This is caused by softening of the in-plane polar modes that become unstable for tensile strains larger than about $2 \%$. Compressive strains larger than $4 \%$ are, instead, necessary to induce ferroelectricity in the direction perpendicular to the strain plane. The strain response is different in the competing ferromagnetic (FM) phase, requiring smaller compressive but larger tensile strains to trigger polar instabilities [17]. At the same time, tensile strain favors the formation of oxygen vacancies, the presence of which was, however, found to be detrimental to ferroelectricity [9].

Fe doping of oxygen-deficient $\left(\mathrm{SrMnO}_{3-\delta}\right)$ thin films could be a strategy to reverse this behavior, since ferroelectricity is expected to be enhanced both by the decrease in crystal symmetry due to the aliovalent $\mathrm{Fe}$ ion and especially by the formation of a defect dipole due to the possible association of the substitutional Fe with an oxygen vacancy [13]. $\mathrm{SrMn}_{1-x} \mathrm{Fe}_{x} \mathrm{O}_{3-\delta}$ was synthesized in the past in an attempt to obtain manganites with a mixture of $d^{3}\left(\mathrm{Mn}^{+4}\right)$ and $d^{4}\left(\mathrm{Fe}^{+4}\right)$ cations [18], as found in other manganites showing colossal magnetoresistance, e.g., the family of doped $\operatorname{Ln}_{1-x}(\mathrm{Ca} / \mathrm{Sr})_{x} \mathrm{MnO}_{3}$ [19,20]. At room temperature, $\mathrm{SrMn}_{1-x} \mathrm{Fe}_{x} \mathrm{O}_{3-\delta}$ phases were found to adopt a cubic perovskite structure with a disordered arrangement of $\mathrm{Mn}$ and Fe transition-metal cations in the octahedral sites due to their similar ionic radii. Oxygen vacancies observed during synthesis in air are generally created to maintain charge balance after the aliovalent substitution and introduce $\mathrm{Fe}^{+3}$ cations in the structure as suggested by iodometric and Mössbauer measurements [18,21,22]. Mössbauer data suggest as well that oxygen vacancies are predominantly found in the vicinity of the $\mathrm{Fe}^{+3}$ ions $[18,21,22]$. $\mathrm{SrMn}_{1-x} \mathrm{Fe}_{x} \mathrm{O}_{3-\delta}$ samples show 


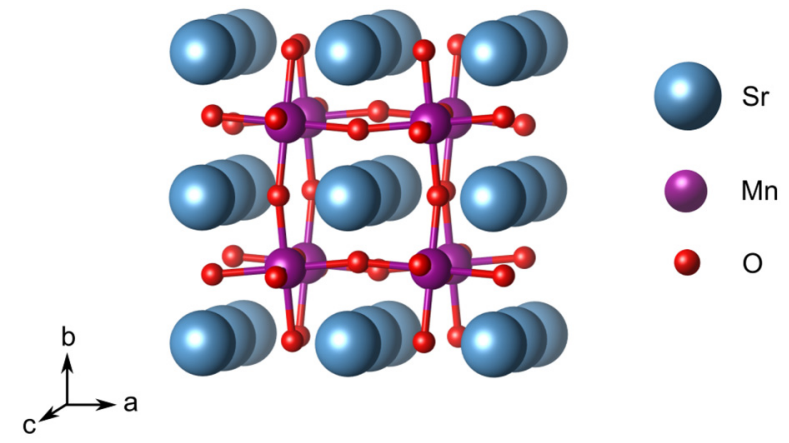

FIG. 1. $(2 \times 2 \times 2)$ Pnma supercell of stoichiometric $\mathrm{SrMnO}_{3}$.

AFM behavior for both low $(x \leqslant 0.3)$ and high $(x \geqslant 0.9)$ Fe doping, while a spin-glass state caused by the interaction between $\mathrm{Fe}^{+3 /+4}$ and $\mathrm{Mn}^{+3 /+4}$ was observed for intermediate dopant concentrations [18,21].

With the aim of understanding the mechanism underlying emerging polarization and the interaction between polar defect pairs, epitaxial strain, and the electronic, structural, and magnetic degrees of freedom, we used density functional theory with a Hubbard $U$ correction $(\mathrm{DFT}+U)$ calculations to investigate the properties of Fe-doped oxygen-deficient SMO thin films. Our results suggest that defect engineering, through controlling the concentration and distribution of polar defect pairs formed by anion vacancies coupled to substitutional cations, constitutes a parameter to design multiferroic materials. Defect couples can, indeed, promote polarity and ferroelectricity in nonpolar perovskites due to an off-center displacement of the defect: the spatially separated substitutional $\mathrm{Fe}_{\mathrm{Mn}}$ (negatively charged) and $\mathrm{V}_{\mathrm{O}}$ (positively charged) offset the charge center from the geometric center of the lattice, resulting in an electric dipole moment along the direction from $\mathrm{Fe}_{\mathrm{Mn}}$ to $\mathrm{V}_{\mathrm{O}}$ already in unstrained and hence nonpolar SMO. Other effects related to the appearance of reduced $\mathrm{Mn}^{+3}$, negatively charged with respect to the $\mathrm{Mn}$ lattice sites, should also be taken into account, since $\mathrm{Mn}^{+3}-\mathrm{V}_{\mathrm{O}}$ pairs can result in additional dipoles that affect the overall ferroelectric response. Finally, the defect-pair dipole can couple with applied epitaxial strain favoring the transition to a polar phase, even for strains lower than the ones necessary to stabilize the polar structure in stoichiometric SMO.

\section{METHODS}

All DFT calculations were performed with the QUANTUM ESPRESSO distribution [23,24]. The revised Perdew-BurkeErnzerhof generalized gradient approximation PBEsol [25] was used as the exchange-correlation functional together with ultrasoft pseudopotentials [26] with $\operatorname{Sr}(4 s, 4 p, 5 s), \operatorname{Mn}(3 p, 4 s$, $3 d)$, and $\mathrm{O}(2 s, 2 p)$ valence states [27]. Kinetic-energy cutoffs of 70 Ry for wave functions and $840 \mathrm{Ry}$ for spin-charge density and potentials were applied. A Gaussian smearing with a broadening parameter of $0.01 \mathrm{Ry}$ was used in all cases.

SMO was simulated as a 40-atom $2 \times 2 \times 2$ supercell of the 5-atom primitive cubic cell. A shifted $6 \times 6 \times 6$ Monkhorst-Pack [28] k-point grid was used to sample the Brillouin zone. Both bulk and thin-film geometries of the

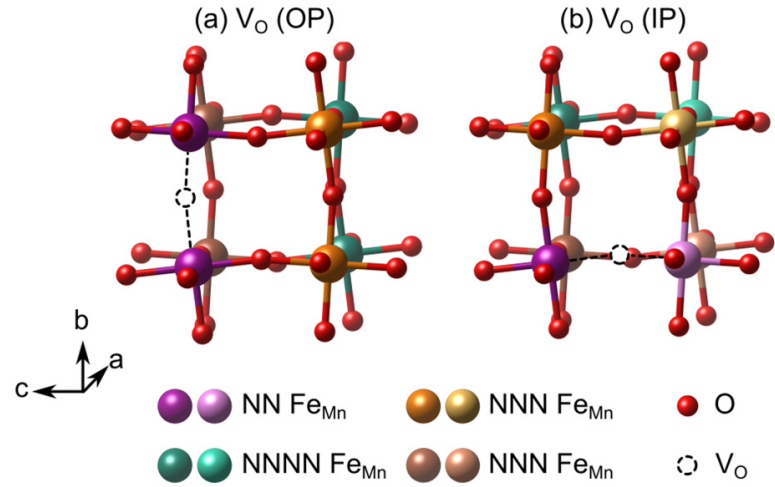

FIG. 2. Schematic representation of the possible relative arrangements of $\mathrm{Fe}_{\mathrm{Mn}}-\mathrm{V}_{\mathrm{O}}$ defect pairs in the 40-atom SMO cell in the case of an (a) out-of-plane (OP) or (b) in-plane (IP) oxygen vacancy. Purple, orange and brown, and green refer to configurations in which $\mathrm{Fe}_{\mathrm{Mn}}$ is in next-neighbor (NN), next-nearest-neighbor (NNN), and next-next-nearest-neighbor (NNNN) position relative to the $\mathrm{V}_{\mathrm{O}}$. As opposed to (a), the two Mn sites at the same distance from the $V_{O}$ in (b) are not symmetry equivalent as indicated by the slightly different shade for each color.

G-type AFM and FM phases of SMO were considered. For stoichiometric bulk calculations, both lattice parameters and atomic positions were relaxed, while thin-film geometries with biaxial epitaxial strain in the $a c$ plane imposed by a cubic substrate were computed following the procedure described in Ref. [29]. Prior to defect creation, the atoms were displaced along the eigenvectors of the polar phonon modes computed for unstrained stoichiometric SMO. Defects were then created by removing one oxygen atom ( $\mathrm{V}_{\mathrm{O}}$, concentration $4.2 \%$ ) and at the same time substituting one $\mathrm{Mn}$ with a $\mathrm{Fe}$ ion $\left(\mathrm{Fe}_{\mathrm{Mn}}\right.$, concentration $\left.12.5 \%\right)$. Different possible relative arrangements of the substitutional $\mathrm{Fe}$ with respect to the $\mathrm{V}_{\mathrm{O}}$ were taken into account (see Fig. 2). Mössbauer experiments have shown that iron is present as $\mathrm{Fe}^{+3}$ when associated with the doubly positively charged $\mathrm{V}_{\mathrm{O}}$ and the substitutional defect is thus negatively charged. The calculations were hence performed considering the positive charge state of this defect pair $\left(\mathrm{Fe}_{\mathrm{Mn}}^{\prime}-\mathrm{V}_{\mathrm{O}}^{\bullet \bullet}\right.$ in Kröger-Vink notation [30], where the prime and dot symbols indicate, respectively, a charge of -1 and +1 relative to the respective lattice site). This charge state results in agreement with experimental observations $[18,21,22]$ of the simultaneous presence of $\mathrm{Fe}^{+3}$ and $\mathrm{Mn}^{+3}$. It was obtained by adjusting the number of electrons and by applying a background charge to ensure neutrality of the unit cell, as required by calculations under periodic boundary conditions to avoid divergences in the electrostatic potential. For simplicity, we will refer to the defect pairs in this charge state simply as $\mathrm{Fe}_{\mathrm{Mn}}-\mathrm{V}_{\mathrm{O}}$. We note that the formation of small self-trapped polarons that was reported for chemically similar systems [31-34] was not considered here. Finally, for defective cells, atomic positions were optimized while keeping the lattice vectors fixed at optimized values of the nondefective system. In all calculations, atomic forces were converged to within $5 \times 10^{-2} \mathrm{eV} / \AA$, while energies were converged to within $1.4 \times 10^{-5} \mathrm{eV}$. Auxiliary calculations using $2 \sqrt{2} \times 2 \sqrt{2} \times 2$, $3 \times 3 \times 3$, and $4 \times 4 \times 4$ supercells with 80,135 , and 320 
atoms (and $3 \times 3 \times 4$ and $3 \times 3 \times 3 \mathbf{k}$ meshes and $\Gamma$-point sampling of the Brillouin zone, respectively) were performed to investigate the interaction of two defect pairs and the influence of the defect concentration on the predicted polarization.

A Hubbard $U$ correction [35-37] was applied in all calculations. For stoichiometric bulk systems, where all Mn sites are crystallographically and chemically equivalent, we used global self-consistent $U$ parameters $U_{\mathrm{SC}}$ computed for the G-AFM and FM phases of SMO in Ref. [38] using densityfunctional perturbation theory (DFPT) [39], as implemented in hp.x of QUANTUM ESPRESSO [23,24]. Self-consistent site-dependent $U$ parameters $U_{\mathrm{SC}-\mathrm{SD}}$ were instead computed for defective systems by perturbing the inequivalent sites resulting from defect formation (atoms were selected to be perturbed if their unperturbed atomic occupations differed by more than $10^{-3}$ ) [38]. DFPT calculations were performed with a $\Gamma$-point sampling of the $\mathbf{q}$ space [39] in the 40-atom cell. A convergence threshold of $0.01 \mathrm{eV}$ was applied for the self-consistence of $U$ values. In all cases, atomic orbitals were used to construct occupation matrices and projectors in the DFT $+U$ scheme. For simplicity, $U_{\mathrm{SC}-\mathrm{SD}}$ values (see Appendix A) have only been computed for the unstrained stoichiometric and defective geometries, since even $4 \%$ tensile strain changes $U_{\mathrm{SC}}$ by only $0.01 \mathrm{eV}$ compared with zero strain [38].

The strain-dependent formation energy $E_{\mathrm{f}}$ of a $\mathrm{Fe}_{\mathrm{Mn}}-\mathrm{V}_{\mathrm{O}}$ defect pair in the $q=+1$ charge state was calculated as described in Ref. [40]:

$$
\begin{aligned}
E_{\mathrm{f}}\left(\epsilon, \mu_{i}, q\right)= & E_{\mathrm{tot}, \mathrm{def}}(\epsilon, q)-E_{\mathrm{tot}, \text { stoic }}(\epsilon) \\
& -\sum_{i} n_{i} \mu_{i}+q E_{\mathrm{Fermi}}(\epsilon)+E_{\mathrm{corr}}(\epsilon) .
\end{aligned}
$$

Here, $E_{\text {tot,def }}$ and $E_{\text {tot,stoic }}$ are the DFT total energies of the defective system with a charge $q$ and of the stoichiometric cell, respectively. $\epsilon$ is the applied strain, and $E_{\mathrm{Fermi}}$ is the Fermi energy relative to the valence band maximum of the defect-free system, which can take values between zero and the band gap of the material; $n_{i}$ indicates the number of atoms of a certain species $i$ that are added $\left(n_{i}>0\right)$ or removed $\left(n_{i}<0\right)$ from the supercell to form the defect, while $\mu_{i}$ is its chemical potential. Finally, $E_{\text {corr }}$ is a corrective term necessary to align the electrostatic potential of the defective cell with that of the neutral stoichiometric system obtained by calculating the difference in electrostatic potential between the neutral defect-free cell and the charged defective one averaged in spheres around atomic sites located far from the defect [41]. No further finitesize corrections were applied since the defect concentrations we simulate are realistic for this material. Different synthesis conditions can be accommodated by adjusting the set of chemical potentials $\mu_{i}=\mu_{i}^{0}+\Delta \mu_{i}$ for each element by assuming equilibrium with a physical reservoir such as a gas or a bulk phase. We expressed $\mu_{\mathrm{Fe}}$ and $\mu_{\mathrm{Mn}}$ as a function of $\mu_{\mathrm{O}}$. For this latter, we used $\mathrm{O}_{2}$ as a reference, $\mu_{\mathrm{O}}=\frac{1}{2} E\left(\mathrm{O}_{2}\right)+\Delta \mu_{\mathrm{O}}$, while bounds on $\Delta \mu_{\mathrm{O}}$ were derived imposing the stability of SMO $\left[\Delta \mu_{\mathrm{Sr}}+\Delta \mu_{\mathrm{Mn}}+3 \Delta \mu_{\mathrm{O}} \leqslant \Delta H_{f}\right.$ (SMO)] against decomposition into elemental $\mathrm{Sr}$ or $\mathrm{Mn}$ species $\left(\Delta \mu_{\mathrm{Sr}} \leqslant\right.$ 0 and $\left.\Delta \mu_{\mathrm{Mn}} \leqslant 0\right)$ and against the formation of competing phases such as $\mathrm{SrO}\left[\Delta \mu_{\mathrm{Sr}}+\Delta \mu_{\mathrm{O}} \leqslant \Delta H_{f}(\mathrm{SrO})\right]$, and $\mathrm{MnO}$ $\left[\Delta \mu_{\mathrm{Mn}}+\Delta \mu_{\mathrm{O}} \leqslant \Delta H_{f}(\mathrm{MnO})\right]$. For the Fe impurity, stability against solubility-limiting phases, such as $\mathrm{FeO}$, were instead considered to relate $\mu_{\mathrm{Fe}}$ to $\mu_{\mathrm{O}}$ [42]. The computed heat of formation $\Delta H_{f}$ of the transition-metal oxides was corrected according to Ref. [43] to account for the mixing of DFT and $\mathrm{DFT}+U$ total energies in the derivation of the formation enthalpy. We will show results in the oxygen-poor limit with $\Delta \mu_{\mathrm{Mn}}=-1.77 \mathrm{eV}$ and $\Delta \mu_{\mathrm{Fe}}=-1.43 \mathrm{eV}$. The Fermi energy was arbitrarily set to the band gap of unstrained SMO, reproducing typical experimental conditions for $n$-doped materials. This choice does not affect our conclusions as we are interested in relative changes of formation energies of different defect configurations and the effect of strain.

The polarization $\vec{P}$ was computed using a point-charge model:

$$
\vec{P}=\sum_{i} \vec{r}_{i} q_{i}
$$

where $\vec{r}_{i}$ is the position of atom $i$ and $q_{i}$ is its nominal charge: +2 for $\mathrm{Sr},-2$ for $\mathrm{O}$, and +4 or +3 for stoichiometric or reduced $\mathrm{Mn}$ and Fe sites. The charge applied on each $\mathrm{Mn}$ and Fe ion was defined on the basis of its oxidation state computed through the method introduced by Sit et al. [44]. The polarization, being a multivalued quantity, has been corrected by an integer number of polarization quanta $\vec{Q}$, computed as

$$
\vec{Q}=\frac{e}{V}\left[\begin{array}{l}
a \\
b \\
c
\end{array}\right],
$$

with $a, b$, and $c$ being the lattice parameters, $V$ being the volume of the unit cell, and $e$ being the elementary charge. Results obtained with this method include polarization contributions of the lattice and the defect dipole but neglect electronic redistribution effects compared with other approaches such as the Berry phase formalism $[45,46]$. However, the metallic nature of some defective SMO cells did not allow the application of the Berry phase approach.

\section{RESULTS AND DISCUSSION}

$\mathrm{SrMn}_{1-x} \mathrm{Fe}_{x} \mathrm{O}_{3-\delta}$ is modeled using all possible symmetryinequivalent $\mathrm{Fe}_{\mathrm{Mn}}-\mathrm{V}_{\mathrm{O}}$ configurations within a $2 \times 2 \times 2 \mathrm{SMO}$ supercell (see Fig. 2). In particular, there are two symmetrydistinct oxygen atoms in this structure: an out-of-plane $\mathrm{V}_{\mathrm{O}}$ with the broken $\mathrm{Mn}-\mathrm{O}-\mathrm{Mn}$ bond perpendicular to the biaxial strain ( $a c$ ) plane [OP; see Fig. 2(a)] and an in-plane O position with the broken $\mathrm{Mn}-\mathrm{O}-\mathrm{Mn}$ bond in the ac plane [IP; see Fig. 2(b)]. For $\mathrm{V}_{\mathrm{O}}^{\mathrm{OP}}$, the $\mathrm{Mn}$ sites lying at nearest-neighbor positions relative to the defect [NN, in purple in Fig. 2(a)] or far away from it [next-next-nearest-neighbor (NNNN) positions, in green in Fig. 2(a)] are equivalent by symmetry, while two different groups of Mn ions can be distinguished for substitution sites in next-nearest-neighbor positions relative to the vacancy [NNN, in orange and brown in Fig. 2(a)] for a total of four symmetry-inequivalent $\mathrm{Fe}_{\mathrm{Mn}}-\mathrm{V}_{\mathrm{O}}^{\mathrm{OP}}$ defect pairs. In contrast, for a $\mathrm{V}_{\mathrm{O}}^{\mathrm{IP}}$, the two Mn atoms lying at $\mathrm{NN}, \mathrm{NNN}$, or NNNN positions correspond each to two symmetry-distinct substitution sites for a total of eight $\mathrm{Fe}_{\mathrm{Mn}}-\mathrm{V}_{\mathrm{O}}^{\mathrm{IP}}$ symmetryinequivalent configurations [see Fig. 2(b)]. 


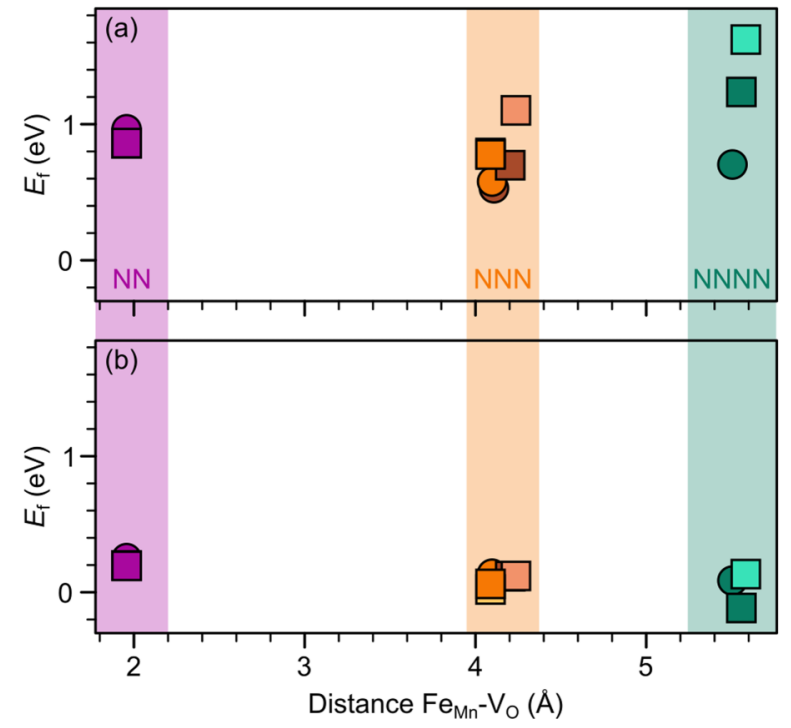

FIG. 3. Formation energy $E_{\mathrm{f}}$ computed for $\mathrm{Fe}_{\mathrm{Mn}}-\mathrm{V}_{\mathrm{O}}$ configurations in unstrained (a) AFM and (b) FM SMO as a function of the $\mathrm{Fe}_{\mathrm{Mn}}-\mathrm{V}_{\mathrm{O}}$ distance. Circles and squares refer to configurations with $\mathrm{V}_{\mathrm{O}}^{\mathrm{OP}}$ and $\mathrm{V}_{\mathrm{O}}^{\mathrm{IP}}$, respectively. See color code in Fig. 2.

In agreement with Mössbauer studies [21], our DFT + $U_{\text {SC-SD }}$ calculations for the insulating AFM phase show a partial reduction of $\mathrm{Mn}$ adjacent to the oxygen vacancy. This implies that for a $\mathrm{Fe}_{\mathrm{Mn}}-\mathrm{V}_{\mathrm{O}}$ defect pair, two dipoles exist in the structure, one pointing from the $\mathrm{Fe}_{\mathrm{Mn}}^{\prime}$ to the $\mathrm{V}_{\mathrm{O}}$ and one pointing from the $\mathrm{Mn}_{\mathrm{Mn}}^{\prime}$ to the $\mathrm{V}_{\mathrm{O}}^{\bullet \bullet}$ as discussed in more detail in Appendix A. This will have implications for the magnetism and polarization as discussed in Secs. III B and III C. In the FM phase a partial reduction of one or two Mn sites is observed, which we associated with its metallic nature.

\section{A. Relative stability and formation energy}

We begin by investigating the relative stability of the $\mathrm{Fe}_{\mathrm{Mn}}-\mathrm{V}_{\mathrm{O}}$ configurations in bulk AFM and FM SMO. We note that we are mainly interested in relative formation-energy differences for the different configurations and in strain-induced changes, rather than absolute defect-pair formation energies, which have been derived in O-poor conditions and thus correspond to a lower limit for $E_{\mathrm{f}}$. In the AFM phase, the most stable configurations are the $\mathrm{NNN} \mathrm{Fe}_{\mathrm{Mn}}-\mathrm{V}_{\mathrm{O}}^{\mathrm{OP}}$, as can be seen from Fig. 3(a), where we report the formation energy $E_{\mathrm{f}}$ computed for the different $\mathrm{Fe}_{\mathrm{Mn}}-\mathrm{V}_{\mathrm{O}}$ arrangements as a function of the distance between the $\mathrm{Fe}_{\mathrm{Mn}}$ and the $\mathrm{V}_{\mathrm{O}}$. The $\mathrm{NN} \mathrm{Fe} \mathrm{Mn}_{\mathrm{Mn}}-\mathrm{V}_{\mathrm{O}}$, the NNNN $\mathrm{Fe}_{\mathrm{Mn}}-\mathrm{V}_{\mathrm{O}}^{\mathrm{OP}}$, and the majority of the $\mathrm{NNN} \mathrm{Fe} \mathrm{Mn}_{\mathrm{O}}-\mathrm{V}_{\mathrm{O}}^{\mathrm{IP}}$ have formation energies higher by about $0.1-0.4 \mathrm{eV}$, while NNNN $\mathrm{Fe}_{\mathrm{Mn}}-\mathrm{V}_{\mathrm{O}}^{\mathrm{IP}}$ configurations are about $0.7-1.0 \mathrm{eV}$ less stable. These results indicate that the $V_{O}$ are preferentially in the neighborhood of the substitutional iron, as suggested by spectroscopic results [22], even though not necessarily in its first coordination shell. More importantly, our data suggest that some disorder is expected, as also indicated by experiments [18]. This is particularly interesting because different configurations correspond to different orientations of the electric dipole associated with the $\mathrm{Fe}_{\mathrm{Mn}}-\mathrm{V}_{\mathrm{O}}$ pair, which-as we
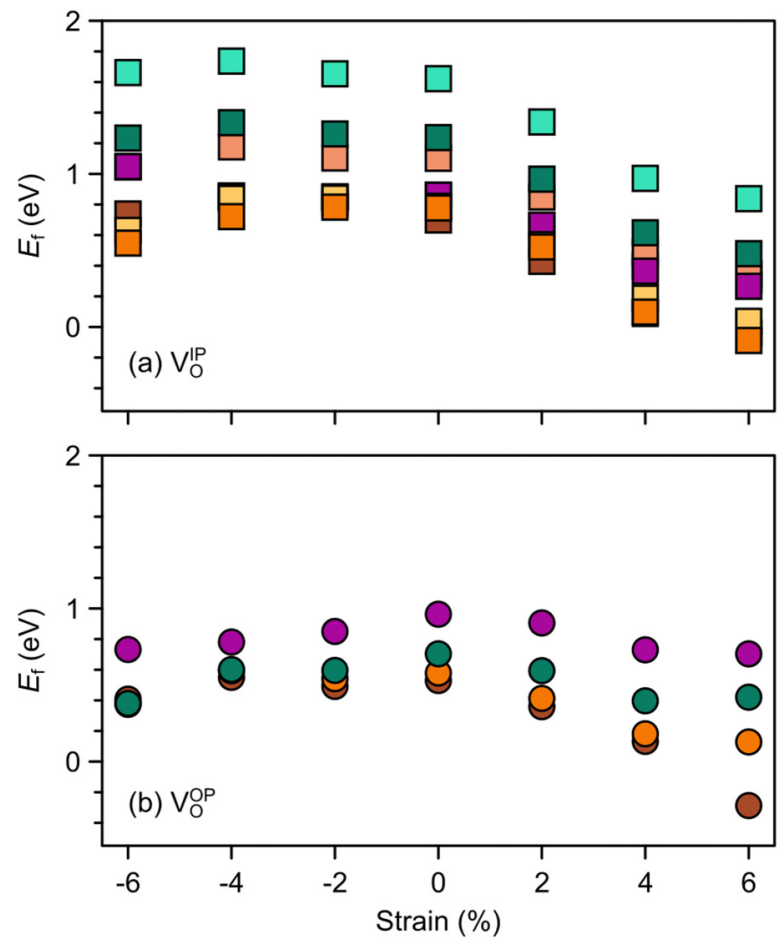

FIG. 4. Strain-dependent formation energy $E_{\mathrm{f}}$ of (a) $\mathrm{Fe}_{\mathrm{Mn}}-\mathrm{V}_{\mathrm{O}}^{\mathrm{IP}}$ and (b) $\mathrm{Fe}_{\mathrm{Mn}}-\mathrm{V}_{\mathrm{O}}^{\mathrm{OP}}$ defect pairs in AFM SMO. See color code in Fig. 2.

will discuss in Sec. III C-is responsible for the polarization in unstrained $\mathrm{SrMn}_{1-x} \mathrm{Fe}_{x} \mathrm{O}_{3-\delta}$. Hence the presence of different energetically similar configurations could potentially lead to a switchable polarization and defect-induced ferroelectricity if the defect dipoles couple in a parallel fashion, which we will explore in Sec. III D.

A different behavior is observed, instead, in the FM phase [see Fig. 3(b)], where not only are $\mathrm{Fe}_{\mathrm{Mn}}-\mathrm{V}_{\mathrm{O}}^{\mathrm{IP}}$ found to be generally more stable regardless of the distance between the substitutional iron and the vacancy, but where the most stable configuration is a NNNN $\mathrm{Fe}_{\mathrm{Mn}}-\mathrm{V}_{\mathrm{O}}^{\mathrm{IP}}$ defect pair, likely because these configurations lead to interactions between $\mathrm{Mn}^{3+}$ and $\mathrm{Mn}^{4+}$ that stabilize the FM phase, as we will discuss in more detail in Sec. III B. Furthermore, the average difference in $E_{\mathrm{f}}$ between the defect configurations in FM SMO is only $0.2 \mathrm{eV}$, and $E_{\mathrm{f}}$ in the FM phase are on average $0.8 \mathrm{eV}$ lower than in the AFM phase. This can be explained by the smaller energetic cost to accommodate the two excess electrons associated with the $\mathrm{V}_{\mathrm{O}}$ on delocalized $\mathrm{Mn}$ or Fe states in the metallic FM phase. In SMO thin films, the defect formation energy and consequently the defect concentration depend on volume changes induced by biaxial strain $[9,48,49]$. Biaxial strain also breaks the symmetry [29] and could thus allow strain-controlled ordering of defects on inequivalent sites $[9,48,49]$. Hence we now consider the interplay between $\mathrm{Fe}_{\mathrm{Mn}}-\mathrm{V}_{\mathrm{O}}$ defects, strain, and magnetism in SMO. Figure 4 shows the changes in $E_{\mathrm{f}}$ for defect pairs as a function of the applied strain. In the AFM phase, tensile strain results in a reduction of the formation energy of $\mathrm{Fe}_{\mathrm{Mn}}-\mathrm{V}_{\mathrm{O}}$, consistent with the chemical expansion [50] due to reduced transition-metal sites. Defect pairs with $\mathrm{V}_{\mathrm{O}}^{\mathrm{IP}}$ are found to be more sensitive to tensile strain: $4 \%$ strain resulting in a reduction of $E_{\mathrm{f}}$ 
by about $0.4-0.7 \mathrm{eV}$ for $\mathrm{Fe}_{\mathrm{Mn}}-\mathrm{V}_{\mathrm{O}}^{\mathrm{IP}}$ compared with only $0.2-$ $0.4 \mathrm{eV}$ for $\mathrm{Fe}_{\mathrm{Mn}}-\mathrm{V}_{\mathrm{O}}^{\mathrm{OP}}$. Consequently, the energy difference between the NNN $\mathrm{Fe}_{\mathrm{Mn}}-\mathrm{V}_{\mathrm{O}}^{\mathrm{OP}}$ and $\mathrm{Fe}_{\mathrm{Mn}}-\mathrm{V}_{\mathrm{O}}^{\mathrm{IP}}$ configurations is reduced, favoring disorder. Under compressive strain, instead, the formation energy of $\mathrm{Fe}_{\mathrm{Mn}}-\mathrm{V}_{\mathrm{O}}$ defect pairs remains fairly constant (average changes of about $0.05 \mathrm{eV}$ ) as already observed for $\mathrm{V}_{\mathrm{O}}$ in SMO because of crystal field effects [48], and $\mathrm{Fe}_{\mathrm{Mn}}-\mathrm{V}_{\mathrm{O}}^{\mathrm{OP}}$ are slightly stabilized with respect to $\mathrm{Fe}_{\mathrm{Mn}}-\mathrm{V}_{\mathrm{O}}^{\mathrm{IP}}$.

Unsurprisingly (as shown in Fig. 19 in Appendix B), the FM phase exhibits a reduced sensitivity of the formation energy to strain, which is rationalized by its metallicity. We also note that for the FM phase, under compressive strain, the formation energy of $\mathrm{Fe}_{\mathrm{Mn}}-\mathrm{V}_{\mathrm{O}}^{\mathrm{IP}}$ defects increases as expected from volume arguments, which can be explained in terms of a reduced sensitivity of the metallic FM phase to crystal field effects, allowing volume effects to dominate [48].

\section{B. Magnetic order}

Bulk stoichiometric SMO has a G-type AFM ground state. DFT $+U$ calculations have shown a $4.2 \%$ oxygen vacancy concentration to induce a magnetic phase transition from AFM to FM $[9,38]$, which is explained by the vacancy-induced $\mathrm{Mn}^{4+}-\mathrm{Mn}^{3+}$ double exchange coupling. The properties of Fe-doped oxygen-deficient SMO are more complex due to the presence of Fe transition-metal atoms. Indeed, for the $\mathrm{Mn}^{4+}, \mathrm{Mn}^{3+}$, and $\mathrm{Fe}^{3+}$ ions present in the simulated cells, the interactions between neighboring $\mathrm{Mn}^{4+}$ and $\mathrm{Mn}^{4+}$ and between neighboring $\mathrm{Mn}^{3+}$ and $\mathrm{Mn}^{3+}$ are AFM, those between $\mathrm{Mn}^{4+}$ and $\mathrm{Mn}^{3+}$ are $\mathrm{FM}$, and those between $\mathrm{Mn}^{4+}$ and $\mathrm{Fe}^{3+}$ are AFM through the $\pi$ orbitals and FM through the $\sigma$ orbitals [18]. Figure 5(a) shows the total energy difference between the unstrained AFM and FM phases with different $\mathrm{Fe}_{\mathrm{Mn}}-\mathrm{V}_{\mathrm{O}}$ defect pairs as a function of the distance between the two defects. We note here that the total energy differences in Fig. 5(a) cannot be directly compared with differences in formation energies shown in Fig. 3. This is due to the fact that the stoichiometric energy, the zero of the Fermi energy (i.e., the valence band edge), and the corrective term appearing in Eq. (1) are different for the two phases and do not cancel when taking formation-energy differences. The most stable configurations (see Fig. 3) favor the AFM order. Indeed, all the configurations with a $\mathrm{V}_{\mathrm{O}}^{\mathrm{OP}}$, and the $\mathrm{NN}$ and the majority of the $\mathrm{NNN} \mathrm{Fe}_{\mathrm{Mn}}-\mathrm{V}_{\mathrm{O}}^{\mathrm{IP}}$ configurations prefer this magnetic phase. Only the NNNN $\mathrm{Fe}_{\mathrm{Mn}}-\mathrm{V}_{\mathrm{O}}^{\mathrm{IP}}$ defects strongly favor the FM phase since the $\mathrm{Mn}^{4+}-\mathrm{Mn}^{3+}$ interactions are promoted due to the larger distance between the $\mathrm{Fe}^{3+}$ ion and the reduced $\mathrm{Mn}^{3+}$ site. This result is in line with the experimental data reporting $\mathrm{SrMn}_{1-x} \mathrm{Fe}_{x} \mathrm{O}_{3-\delta}$, with $\mathrm{Fe}$ concentration close to the concentration in our study $(x=0.125)$, to show AFM behavior. Interestingly, the DFT $+U_{\mathrm{SC}-\mathrm{SD}}$ approach including local chemical changes on the transition-metal atoms around the defect is fundamental to predict defect-induced magnetic properties, since DFT $+U_{\mathrm{SC}}$ with global $U_{\mathrm{SC}}$ of stoichiometric SMO predicts a preferential FM order for all configurations (see Fig. 20 in Appendix C). This result can be explained by increasing $U$ favoring the FM order, which conversely implies that the decreased $U_{\text {SC-SD }}$ values on the reduced $\mathrm{Mn}^{3+}$ will locally destabilize the FM order [38].
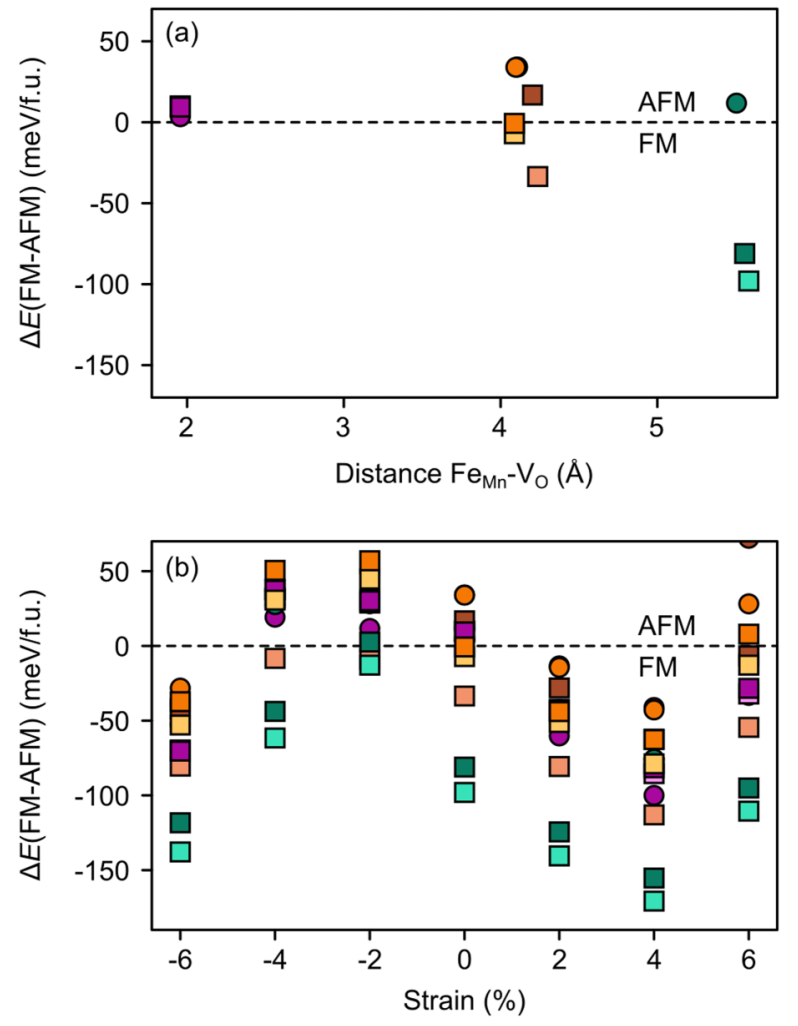

FIG. 5. Total energy differences $[\Delta E$ (FM-AFM)] per formula unit between the defective cells with FM and AFM order. AFM is more stable for positive differences, and FM is more stable for negative differences. (a) $\Delta E$ (FM-AFM) reported with respect to the $\mathrm{Fe}_{\mathrm{Mn}}-\mathrm{V}_{\mathrm{O}}$ distance in each defective configuration in the unstrained SMO structure. (b) Changes in $\Delta E$ (FM-AFM) as a function of strain for all the considered configurations. Circles and squares refer to data obtained for $\mathrm{V}_{\mathrm{O}}^{\mathrm{OP}}$ and $\mathrm{V}_{\mathrm{O}}^{\mathrm{IP}}$, respectively. See color code in Fig. 2.

Biaxial strain beyond a critical value of $2 \%$ is known to induce an AFM-to-FM transition in stoichiometric SMO [9,38]. We now consider the interplay between this magnetic phase transition and the $\mathrm{Fe}_{\mathrm{Mn}}-\mathrm{V}_{\mathrm{O}}$ defect pair [see Fig. 5(b)]. Unsurprisingly, tensile strain stabilizes the FM phase even in the presence of the defect pair, affecting all configurations in almost the same way and in an approximately linear fashion up to $4 \%$ strain. Larger tensile strain results, instead, in a stabilization of the AFM phase for the most stable configurations and in a reduction of the FM stabilization for the others. This observation can be explained considering the stronger sensitivity of the AFM phase to volume changes, which results, as previously discussed, in a larger reduction of the formation energy and consequently in a stabilization of the AFM phase for large tensile strains. Compressive strain up to $-2 \%$ favors the AFM order: At $-2 \%$ the majority of the configurations show an AFM ground state, and for the remaining cases the two magnetic orders are very close in energy. The preference for the AFM order, instead, decreases for larger compressive strain due to the increased stability of the FM phase in compressively strained stoichiometric SMO films [38]. 

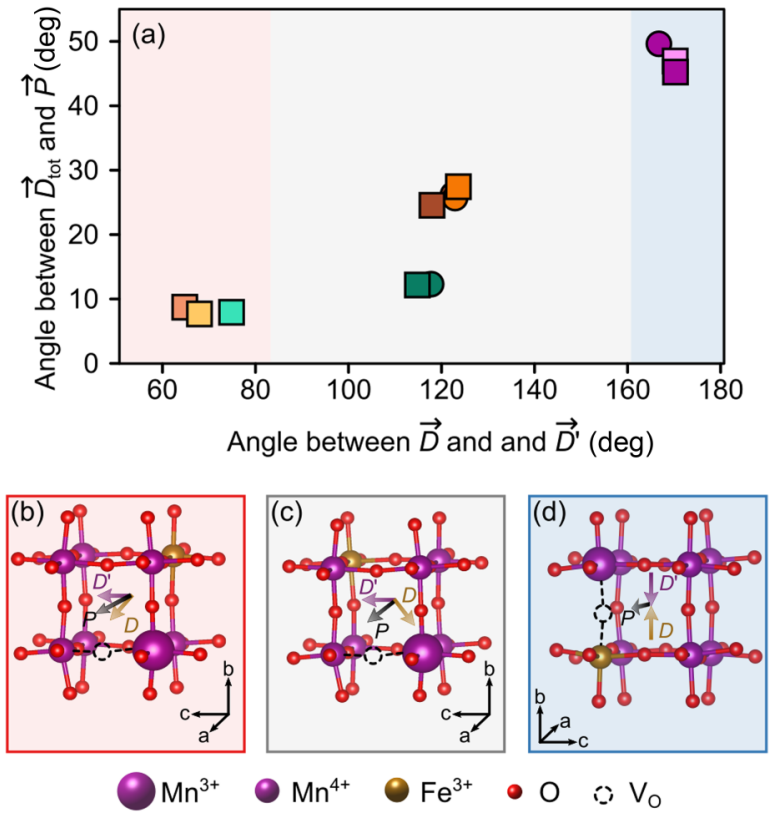

FIG. 6. (a) Angle between the total dipole $\vec{D}_{\text {tot }}$ and the computed polarization $\vec{P}$ with respect to the angle between the $\mathrm{Fe}_{\mathrm{Mn}}^{\prime}-\mathrm{V}_{\mathrm{O}}^{\bullet \bullet}$ defect dipole $\vec{D}$ and the $\mathrm{Mn}_{\mathrm{Mn}}^{\prime}-\mathrm{V}_{\mathrm{O}}^{\bullet \bullet}$ dipole $\vec{D}^{\prime}$ for the different defect-pair configurations. Circles and squares refer to data obtained for $\mathrm{V}_{\mathrm{O}}^{\mathrm{OP}}$ and $\mathrm{V}_{\mathrm{O}}^{\mathrm{IP}}$, respectively. See color code in Fig. 2. (b)-(d) Schematic representation of the $\vec{D}, \vec{D}^{\prime}$, and $\vec{P}$ vectors for the cases in which the angle between $\vec{D}$ and $\vec{D}^{\prime}$ is about $60^{\circ}$ (b), $120^{\circ}$ (c), or $180^{\circ}$ (d).

\section{Polarization in unstrained $\mathrm{SrMn}_{1-x} \mathrm{Fe}_{x} \mathrm{O}_{3-\delta}$}

We now consider the polarization in Fe-doped oxygendeficient SMO by starting from the unstrained geometries. Polarization can arise due to symmetry reduction by the defect pair but also due to the formation of a defect dipole $\vec{D}$ : The spatially separated substitutional $\mathrm{Fe}_{\mathrm{Mn}}^{\prime}$ (negatively charged) and $\mathrm{V}_{\mathrm{O}}^{\bullet \bullet}$ (positively charged) result in the charge center being offset from the geometric center of the lattice and hence an electric dipole moment along the direction from $\mathrm{Fe}_{\mathrm{Mn}}$ to $\mathrm{V}_{\mathrm{O}}$ [13]. As detailed in Appendix A, the situation is further complicated in the AFM phase by the reduction of one $\mathrm{Mn}$ ion $\left(\mathrm{Mn}_{\mathrm{Mn}}^{\prime}\right)$ adjacent to $\mathrm{V}_{\mathrm{O}}$, which induces an additional defect dipole $\vec{D}^{\prime}$ from the negatively charged reduced $\mathrm{Mn}_{\mathrm{Mn}}^{\prime}$ to the positively charged $\mathrm{V}_{\mathrm{O}}^{\bullet}$. For simplicity, we will concentrate the following discussion mainly on results obtained for the AFM order. Indeed, the metallic nature of the FM phase and the consequent partial reduction of more than one Mn (cf. Appendix A) result in a more complex behavior, which may not be properly described within the simple approach we use to estimate the polarization based on nominal charges (see Appendix D). Results for the FM phase are reported in Appendix D 2.

The computed polarization $\vec{P}$ is roughly aligned with the vector sum $\vec{D}+\vec{D}^{\prime}=\vec{D}_{\text {tot }}$, forming with $\vec{D}_{\text {tot }}$ an angle ranging from about $6^{\circ}$ to $50^{\circ}$ for the different configurations as shown in Fig. 6(a). The alignment between $\vec{D}_{\text {tot }}$ and $\vec{P}$ depends on the relative geometric arrangement of $\mathrm{Fe}_{\mathrm{Mn}}^{\prime}, \mathrm{Mn}_{\mathrm{Mn}}^{\prime}$, and $\mathrm{V}_{\mathrm{O}}$. In particular, due to geometric constraints, the angle between $\vec{D}$ and $\vec{D}^{\prime}$ can be around either $60^{\circ}$ [see Fig. 6(b)], $120^{\circ}$ [see Fig. 6(c)], or $180^{\circ}$ [see Fig. 6(d)]. The smaller this angle, the
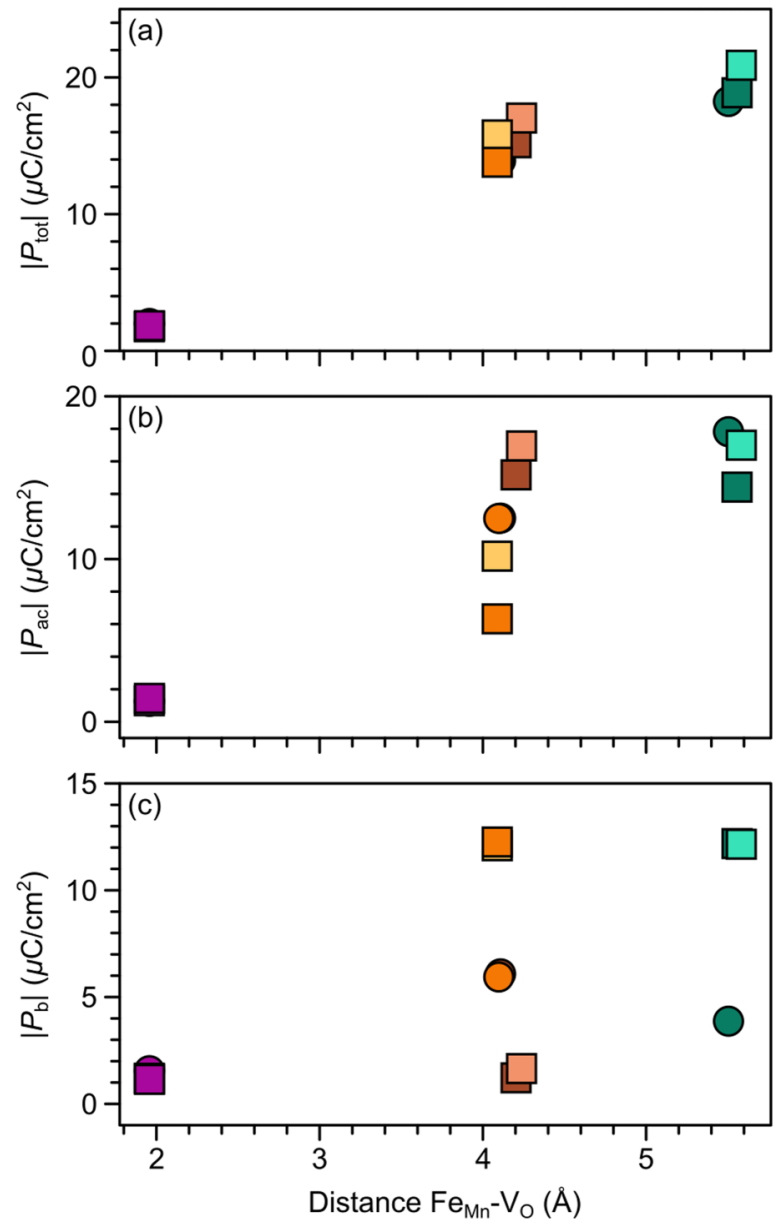

FIG. 7. Magnitude of (a) the total polarization vector, as well as its (b) in-plane $\left(\left|P_{a c}\right|\right)$ and (c) out-of-plane $\left(\left|P_{b}\right|\right)$ components for the different defect configurations in the AFM phase. Circles and squares refer to data obtained for $\mathrm{V}_{\mathrm{O}}^{\mathrm{OP}}$ and $\mathrm{V}_{\mathrm{O}}^{\mathrm{IP}}$, respectively. See color code in Fig. 2.

stronger the combination of the two dipoles and consequently the larger the alignment between $\vec{P}$ and $\vec{D}_{\text {tot }}$ [see Fig. 6(a) and also Appendix D]. For example, for $\mathrm{NN} \mathrm{Fe}_{\mathrm{Mn}^{-}}-\mathrm{V}_{\mathrm{O}}$ configurations, the $\mathrm{Fe}_{\mathrm{Mn}}^{\prime}$ and $\mathrm{Mn}_{\mathrm{Mn}}^{\prime}$ ions are located at the two sites adjacent to $\mathrm{V}_{\mathrm{O}}$, resulting in antiparallel $\vec{D}$ and $\vec{D}^{\prime}$ dipoles [see Fig. 6(d)]. As a result of this peculiar arrangement of the defect dipoles and of the smaller distance between the substitutional iron and the vacancy, $\mathrm{NN} \mathrm{Fe}_{\mathrm{Mn}}-\mathrm{V}_{\mathrm{O}}$ configurations are associated with the smallest total polarization $P_{\text {tot }}$ of about 2 $\mu \mathrm{C} / \mathrm{cm}^{2}$ [see Fig. 7(a)]. This value is close to the one obtained for an isolated $\mathrm{V}_{\mathrm{O}}^{\bullet}$ in SMO $\left(1.90 \mu \mathrm{C} / \mathrm{cm}^{2}\right)$, where the two extra electrons reduce the two $\mathrm{Mn}$ adjacent to the defect, resulting in two largely canceling $\mathrm{Mn}^{\prime}-\mathrm{V}_{\mathrm{O}}^{\bullet \bullet}$ dipoles. Unsurprisingly, $P_{\text {tot }}$ slightly increases with increasing $\mathrm{Fe}_{\mathrm{Mn}}-\mathrm{V}_{\mathrm{O}}$ distance up to $15-20 \mu \mathrm{C} / \mathrm{cm}^{2}$ [see Fig. 7(a)], showing the importance of doping with $\mathrm{Fe}$ to form $\mathrm{Fe}_{\mathrm{Mn}}-\mathrm{V}_{\mathrm{O}}$ defect pairs with associated large polarization. Indeed, these polarizations are of similar magnitude to the ones in conventional ferroelectrics such as $\mathrm{BaTiO}_{3}$ [51]. The in-plane component of the polarization $\left[P_{a c}\right.$; see Fig. 7(b)] behaves similarly and is generally larger than the out-of-plane component $P_{b}$ [see Fig. 7(c)]. $P_{b}$ reflects, instead, the relative arrangement of the $\mathrm{Fe}_{\mathrm{Mn}}$ and $\mathrm{V}_{\mathrm{O}}$ 

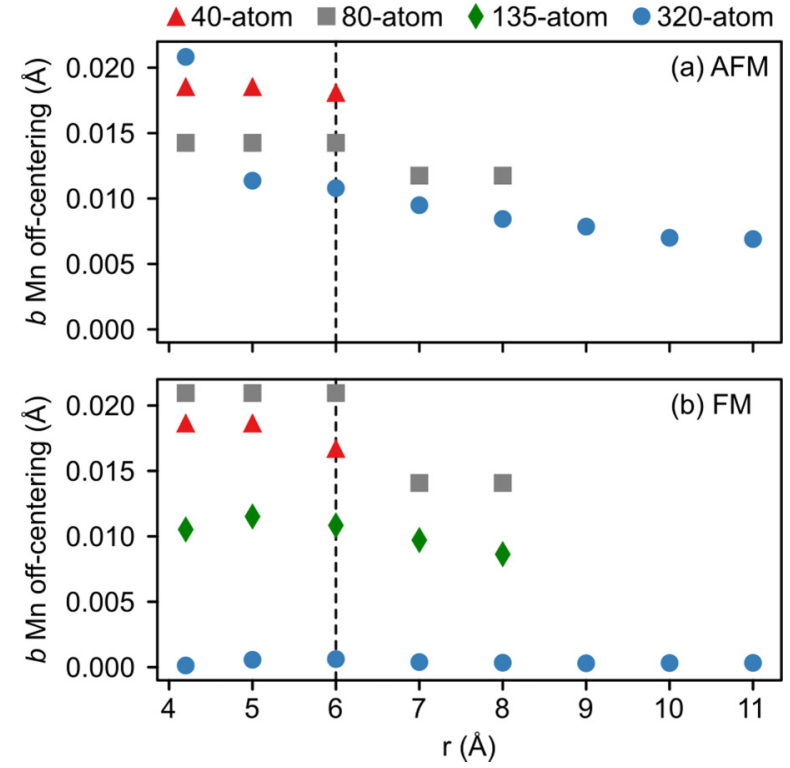

FIG. 8. Total off-centering along the $b$ axis for Mn atoms lying within a sphere of radius $r$ and centered on the center of mass of the defect pair. Results are for different cell sizes in both unstrained (a) AFM and (b) FM SMO.

defects along the $b$ axis: For NNN $\mathrm{Fe}_{\mathrm{Mn}}-\mathrm{V}_{\mathrm{O}}^{\mathrm{IP}}$ configurations, the defects can belong to the same atomic layer [see configurations in brown in Fig. 2(b)] showing $P_{b}$ values lower than $1 \mu \mathrm{C} / \mathrm{cm}^{2}$, or they can belong to atomic layers separated by about $3.8 \AA$ along $b$ [see configurations in orange and green in Fig. 2(b)] with $P_{b}$ values of about $12 \mu \mathrm{C} / \mathrm{cm}^{2}$. NNN and $\mathrm{NNNN} \mathrm{Fe}_{\mathrm{Mn}}-\mathrm{V}_{\mathrm{O}}^{\mathrm{OP}}$ have instead intermediate $P_{b}$ values of about $8-10 \mu \mathrm{C} / \mathrm{cm}^{2}$ since $\mathrm{Fe}_{\mathrm{Mn}}$ and $\mathrm{V}_{\mathrm{O}}$ defects always belong to atomic planes at a distance of about $1.9 \AA$.

These results suggest that the mechanism underlying the observed polarization in unstrained SMO is the off-centering of the charge due to the separation of the $\mathrm{Fe}_{\mathrm{Mn}}^{\prime}$ or $\mathrm{Mn}_{\mathrm{Mn}}^{\prime}$ and the $\mathrm{V}_{\mathrm{O}}^{\bullet \bullet}$ defects.

\section{Polarization and defect concentration}

In order to further investigate the mechanism underlying the polarization induced by the defect pair, we now examine how the defect concentration impacts the polarization of Fe-doped oxygen-deficient SMO. Figure 23 in Appendix D shows that the polarization decreases with increasing cell size (i.e., with decreasing the defect concentration). This suggests the polarization to originate from a local change in symmetry around the defect pair due to the defect dipoles. Indeed, $\mathrm{Fe}_{\mathrm{Mn}}-\mathrm{V}_{\mathrm{O}}$ defects induce small displacements of the atoms in the vicinity of the defect pair from their highsymmetry positions. For example, Fig. 8 shows the Mn displacements along the $b$ axis (with the largest polarization component) for the defect configuration considered in Fig. 23 in Appendix D and computed in supercells of different size. These off-centerings have been computed excluding the two $\mathrm{Mn}$ adjacent to the oxygen vacancy to avoid artifacts due to the relaxation of these undercoordinated sites. In general, the larger the polarization in Fig. 23 in Appendix D, the larger the Mn off-centerings. More importantly, the Mn displacements (a)
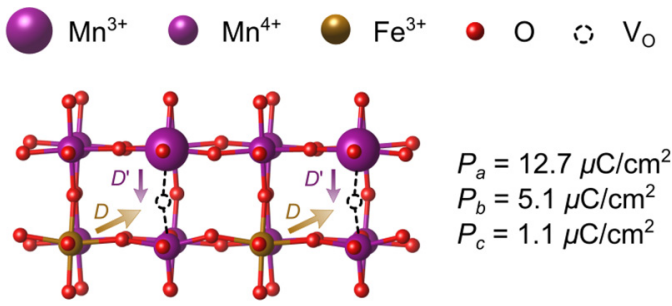

(b)

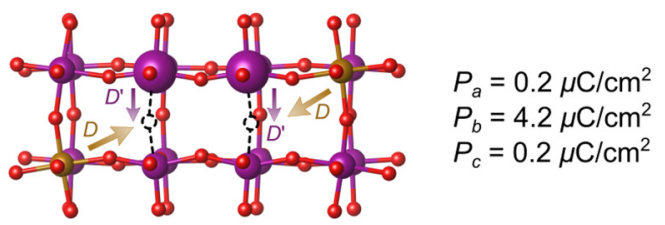

(c)
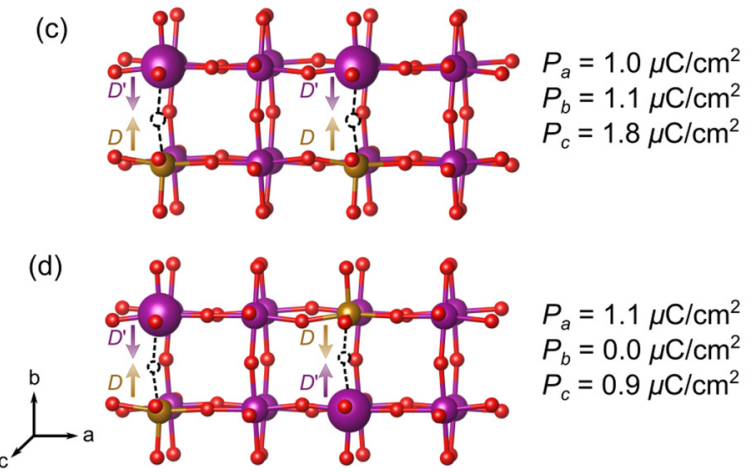

FIG. $9.4 \times 2 \times 2$ supercells with two aligned [(a) and (c)] and antialigned [(b) and (d)] $\mathrm{Fe}_{\mathrm{Mn}}-\mathrm{V}_{\mathrm{O}}$ defect pairs. Illustrations are for (a) and (b) NNN and (c) and (d) $\mathrm{NN} \mathrm{Fe}_{\mathrm{Mn}}-\mathrm{V}_{\mathrm{O}}^{\mathrm{OP}}$. Gold and purple arrows indicate the direction of the $\mathrm{Fe}_{\mathrm{Mn}}^{\prime}-\mathrm{V}_{\mathrm{O}}^{\bullet \bullet}$ and $\mathrm{Mn}_{\mathrm{Mn}}^{\prime}-\mathrm{V}_{\mathrm{O}}^{\bullet \bullet}$ defect dipoles, respectively.

are generally larger and constant for sites lying within $6 \AA$ from the defect pair and decrease afterwards, pointing to a spatially limited effect of the defect pair.

High defect concentrations may thus promote macroscopic polarization, but the possibility of different orientations of neighboring defect dipoles should be taken into account. For this reason, we performed additional calculations in a $4 \times 2 \times 2$ supercell containing two $\mathrm{Fe}_{\mathrm{Mn}}-\mathrm{V}_{\mathrm{O}}$ defect pairs and investigated the cases in which the $\mathrm{Fe}_{\mathrm{Mn}}-\mathrm{V}_{\mathrm{O}}$ defect dipoles lie parallel or antiparallel to one another. Due to the importance of elastic effects for defects in close proximity [5,52], both atomic positions and lattice parameters were allowed to relax in these calculations.

Figure 9 schematically illustrates the structures and orientation of the defect dipoles for the most stable $\mathrm{NNN} \mathrm{Fe}_{\mathrm{Mn}}-\mathrm{V}_{\mathrm{O}}^{\mathrm{OP}}$ configuration, in which the defect dipoles lie mainly in the ac plane [Figs. 9(a) and 9(b)], and for the most stable $\mathrm{NN} \mathrm{Fe}_{\mathrm{Mn}}-\mathrm{V}_{\mathrm{O}}^{\mathrm{OP}}$ configuration, in which the defect dipoles lie mainly along the $b$ axis [Figs. 9(c) and 9(d)]. In order to explain the obtained polarization in the presence of the two parallel or antiparallel dipoles, the orientation of both the $\mathrm{Fe}_{\mathrm{Mn}}^{\prime}-\mathrm{V}_{\mathrm{O}}^{\bullet \bullet}$ and $\mathrm{Mn}_{\mathrm{Mn}}^{\prime}-\mathrm{V}_{\mathrm{O}}^{\bullet \bullet}$ defect dipoles has to be considered. As discussed in Sec. III C, for configurations such as NN $\mathrm{Fe}_{\mathrm{Mn}}-\mathrm{V}_{\mathrm{O}}^{\mathrm{OP}}$, in which the substitutional $\mathrm{Fe}$ is adjacent to $\mathrm{V}_{\mathrm{O}}$, the resulting polarization lies almost along the direction given by the combination of the $\mathrm{Fe}_{\mathrm{Mn}}^{\prime}-\mathrm{V}_{\mathrm{O}}^{\bullet \bullet}$ and $\mathrm{Mn}_{\mathrm{Mn}}^{\prime}-\mathrm{V}_{\mathrm{O}}^{\bullet \bullet}$ and has 
nearly equal components along the $a, b$, and $c$ directions [see Fig. 9(c)]. Introducing two antiparallel $\mathrm{NN} \mathrm{Fe} \mathrm{Mn}-\mathrm{V}_{\mathrm{O}}^{\mathrm{OP}}$ results in two opposite $\mathrm{Fe}_{\mathrm{Mn}}^{\prime}-\mathrm{V}_{\mathrm{O}}^{\bullet \bullet}$ and $\mathrm{Mn}_{\mathrm{Mn}}^{\prime}-\mathrm{V}_{\mathrm{O}}^{\bullet \bullet}$ dipoles along the $b$ axis, and consequently in quenching the polarization component along this axis [see Fig. 9(d)]. In contrast, for the NNN $\mathrm{Fe}_{\mathrm{Mn}}-\mathrm{V}_{\mathrm{O}}^{\mathrm{OP}}$ configuration, the polarization is larger along $a$ due to the longer $\mathrm{Fe}_{\mathrm{Mn}}^{\prime}-\mathrm{V}_{\mathrm{O}}^{\bullet \bullet}$ dipole with a smaller component along $b$ also due to the $\mathrm{Mn}_{\mathrm{Mn}}^{\prime}-\mathrm{V}_{\mathrm{O}}^{\bullet \bullet}$ dipole [see Fig. 9(a)]. Two antiparallel $N N N \mathrm{Fe}_{\mathrm{Mn}}-\mathrm{V}_{\mathrm{O}}^{\mathrm{OP}}$ defect pairs result in quenching the large component of the polarization along $a$, but in only a small decrease along $b$, due to the fact that the $\mathrm{Mn}_{\mathrm{Mn}}^{\prime}-\mathrm{V}_{\mathrm{O}}^{\bullet \bullet}$ dipoles are still aligned along this axis [see Fig. 9(b)]. In both cases, the parallel alignment of the defect dipoles is energetically favored by $0.02 \mathrm{eV}$ for the $\mathrm{NN} \mathrm{Fe} \mathrm{Mn}_{\mathrm{O}}-\mathrm{V}_{\mathrm{O}}^{\mathrm{OP}}$ and even more (by $0.26 \mathrm{eV}$ ) for $\mathrm{NNN} \mathrm{Fe}_{\mathrm{Mn}}-\mathrm{V}_{\mathrm{O}}^{\mathrm{OP}}$, suggesting a parallel coupling of the defect dipoles even at room temperature.

Alignment of the defect dipoles is only one of the two requirements for defect-induced ferroelectricity, switchability of the defect dipoles via an electric field being the other. In Ref. [17], we showed that, for $\mathrm{V}_{\mathrm{Sr}}-\mathrm{V}_{\mathrm{O}}$ defect pairs in SMO, polarization switching occurs via diffusion of oxygen vacancies. This process is associated with barriers of about $0.7 \mathrm{eV}$, which we expect not to be largely altered in the present case, and which allow polarization reversal via the application of electric fields of reasonable magnitude. We thus expect that oxygen diffusion can result in defect-dipole switching also for $\mathrm{Fe}_{\mathrm{Mn}}^{\prime}-\mathrm{V}_{\mathrm{O}}^{\bullet \bullet}$ defect pairs.

\section{E. Interplay between polarization and strain}

It is established that epitaxial strain imposed, for example, by lattice matching with a substrate during coherent epitaxial growth of thin films breaks the symmetry and affects competing energy contributions. Hence it constitutes a viable strategy to induce ferroelectric properties in nonpolar oxides $[8,12]$. In this section, we discuss how the $\mathrm{Fe}_{\mathrm{Mn}}-\mathrm{V}_{\mathrm{O}}$ defect chemistry interacts with strain and with the magnetic properties in determining the polar properties of SMO thin films.

For tensile-strained AFM Fe-doped oxygen-deficient SMO, we observe a general increase in the in-plane components of the polarization $\left(P_{a}\right.$ and $P_{c}$; see Fig. 10): at $4 \%$ strain by about $7 \mu \mathrm{C} / \mathrm{cm}^{2}$ for $P_{a}$ and $12(7) \mu \mathrm{C} / \mathrm{cm}^{2}$ for $P_{c}$ for $\mathrm{Fe}_{\mathrm{Mn}}-\mathrm{V}_{\mathrm{O}}^{\mathrm{OP}}\left(\mathrm{Fe}_{\mathrm{Mn}}-\mathrm{V}_{\mathrm{O}}^{\mathrm{IP}}\right)$ defects. This increase in polarization is accompanied by an average increase in the Mn off-centering by about 0.07 and $0.15 \AA$ at $+4 \%$ strain along the $a$ and $c$ axis, respectively [see Figs. 11(a) and 11(b) and Figs. 11(e) and 11(f)]. Conversely, compressive strain is associated with an increase [up to $3 \mu \mathrm{C} / \mathrm{cm}^{2}$; see Figs. 10 (c) and 10 (d)] in the out-of-plane component of the polarization associated with Mn off-centerings of about $0.03-0.1 \AA$ along the $b$ axis [see Figs. 11(c) and 11(d)] already for $-4 \%$ strain. Interestingly, this strain is smaller than the $-6 \%$ predicted to be necessary to destabilize the polar out-of-plane phonon mode in AFM SMO [17]. Indeed, the larger Mn displacements computed in the presence of defect pairs compared with the stoichiometric case (see Fig. 11) in a strain range between -4 and $2 \%$ further highlight the ability of defect pairs to favor the polar phase transition.

Interestingly, in the FM phase, polarization was found to be roughly constant as a function of the applied strain (cf.
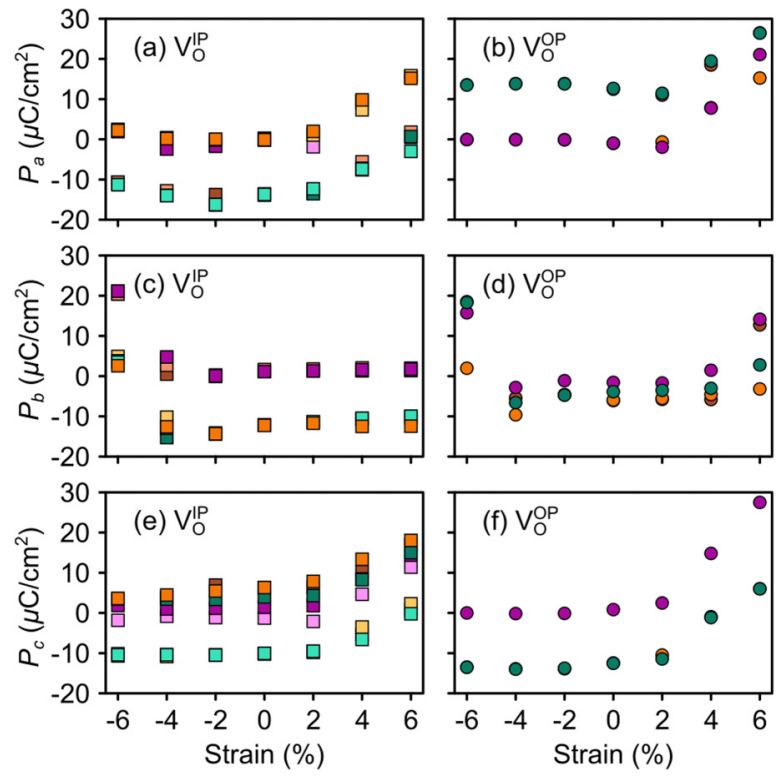

FIG. 10. Strain dependence of the $a$ [(a) and (b)], $b$ [(c) and (d)], and $c[(e)$ and (f)] components of the polarization for the different defect-pair configurations in AFM SMO. Plots are for (a), (c), and (e) $\mathrm{Fe}_{\mathrm{Mn}}-\mathrm{V}_{\mathrm{O}}^{\mathrm{OP}}$ and (b), (d), and (f) $\mathrm{Fe}_{\mathrm{Mn}}-\mathrm{V}_{\mathrm{O}}^{\mathrm{IP}}$ defect pairs. Circles and squares refer to data obtained for $\mathrm{V}_{\mathrm{O}}^{\mathrm{OP}}$ and $\mathrm{V}_{\mathrm{O}}^{\mathrm{IP}}$, respectively. See color code in Fig. 2.

Fig. 24 in Appendix D), between -2 and $4 \%$ strain with small Mn off-centerings of the same magnitude as in the unstrained structure (cf. Fig. 25 in Appendix D). A smaller increase in $P_{b}$ $\left(P_{a c}\right)$ and in the Mn off-centerings along the $b$ axis ( $a c$ plane) starting for a strain of about $-4 \%(6 \%)$ is observed also in the FM phase. The different behavior of the FM phase can be explained by the larger electronic screening of the defect dipole in the metallic FM phase and the strain dependence of the polar modes in stoichiometric SMO, where the IP modes soften only for large tensile strains beyond $6 \%$ and the OP mode becomes unstable at $2 \%$ compressive strain [17].

In summary, not only do these results suggest that doping SMO thin films with Fe can reverse the suppression of the ferroelectricity by oxygen vacancies generally present in the samples [9], but also they suggest that $\mathrm{Fe}_{\mathrm{Mn}}-\mathrm{V}_{\mathrm{O}}$ defect pairs can, depending on the magnetic order, couple with strain to favor the polar phase transition.

\section{CONCLUSIONS}

In this paper we used DFT $+U_{\text {SC-SD }}$ calculations to investigate the potential of inducing ferroelectricity in $\mathrm{SrMnO}_{3}$ (SMO) through polar defect pairs, formed by a substitutional $\mathrm{Fe}$ atom and an oxygen vacancy. We further studied the interplay of these defect pairs with epitaxial strain and the magnetic phase. DFT $+U_{\mathrm{SC}-\mathrm{SD}}$ is fundamental to describe electronic-structure changes upon defect formation and to reconcile predicted magnetic properties with the available experimental data.

Our results suggest that defect engineering via polar defect pairs constitutes a parameter to design materials where two ferroic orders coexist. Investigation of the coupling between 

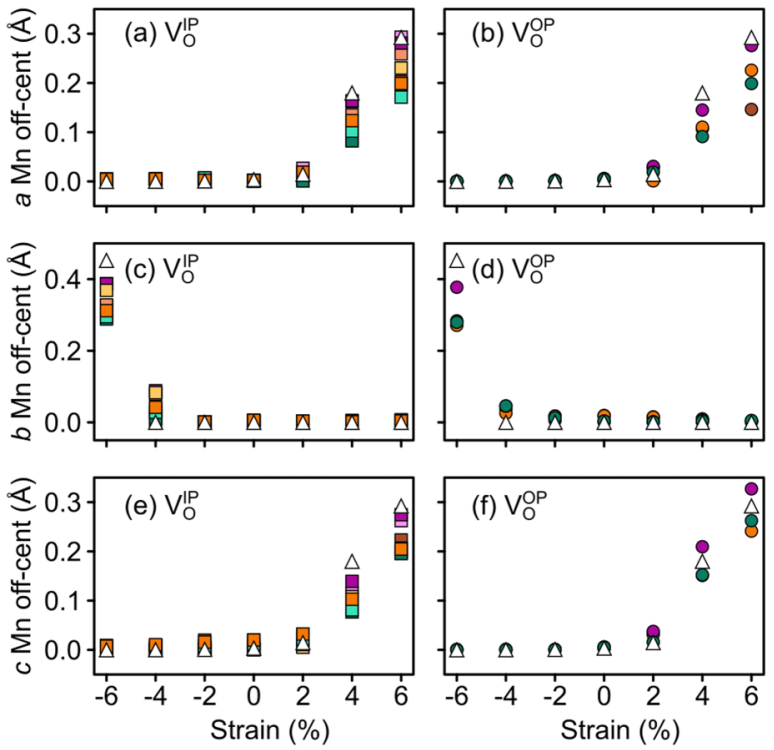

FIG. 11. Strain dependence of the average Mn off-centering along the $a$ axis [(a) and (b)], $b$ axis [(c) and (d)], and $c$ axis [(e) and (f)] for the different defect-pair configurations in the AFM phase of SMO. Plots are for (a), (c), and (e) $\mathrm{Fe}_{\mathrm{Mn}}-\mathrm{V}_{\mathrm{O}}^{\mathrm{OP}}$ and (b), (d), and (f) $\mathrm{Fe}_{\mathrm{Mn}}-\mathrm{V}_{\mathrm{O}}^{\mathrm{IP}}$ defects. Circles and squares refer to data obtained for $\mathrm{V}_{\mathrm{O}}^{\mathrm{OP}}$ and $\mathrm{V}_{\mathrm{O}}^{\mathrm{IP}}$, respectively. See color code in Fig. 2. The white triangles correspond, instead, to the Mn off-centerings in the stoichiometric SMO cells.

these orders, which determines them to be type-I or type-II multiferroics [53], is needed. Ferroelectricity in nominally nonpolar SMO can arise due to an off-center displacement of the defect charge resulting in a net electric dipole moment along the direction from the negatively charged substitutional $\mathrm{Fe}_{\mathrm{Mn}}^{\prime}$ and $\mathrm{Mn}_{\mathrm{Mn}}^{\prime}$ sites to the positively charged $\mathrm{V}_{\mathrm{O}}$. Furthermore, the defect pairs lead to a small off-centering of the Mn atoms in the defect neighborhood from their high-symmetry positions.

Epitaxial strain can couple with $\mathrm{Fe}_{\mathrm{Mn}}-\mathrm{V}_{\mathrm{O}}$, not only reducing the defect formation energy (and hence increasing the defect concentration) under tensile strain but also, more importantly, inducing polarity either in plane or out of plane for tensile and compressive strain, respectively, already for strains smaller than those required to induce a polar phase transition in the defect-free material.

These results and the fact that local defect-induced dipoles couple in a parallel fashion establish polar defect pairs as a promising route to engineer ferroelectricity in nominally nonpolar transition-metal oxides.

All data is available on the Materials Cloud at Ref. [47].

\section{ACKNOWLEDGMENTS}

This research was supported by the NCCR MARVEL, funded by the Swiss National Science Foundation. Computational resources were provided by the University of Bern (on the HPC cluster UBELIX [54]), by the Swiss National Supercomputing Center (CSCS) under project ID mr26, and by SuperMUC at GCS@LRZ, Germany, for which we acknowledge PRACE for awarding us access.

\section{APPENDIX A: SELF-CONSISTENT SITE-DEPENDENT DFT $+U_{\text {SC-SD }}$}

\section{Hubbard correction for Mn: $U_{M n}$}

Defect formation in transition-metal materials can result in local perturbations of the chemical environment of Hubbard sites around the defect, upon which the Hubbard parameters physically depend. For this reason, we recently proposed a self-consistent and site-dependent $\mathrm{DFT}+U_{\mathrm{SC}-\mathrm{SD}}$ approach in which the $U$ values are computed for all inequivalent Hubbard sites [38]. DFT $+U_{\mathrm{SC}-\mathrm{SD}}$ was found to be promising to predict properties of defective systems, in particular, when excesscharge localization is restricted to atoms around the defect site and can be properly captured by site-dependent $U$ values. For example, in the case of $\mathrm{V}_{\mathrm{O}}$ in $\mathrm{SMO}, U$ values were found to depend on the distance of the Hubbard site from the defect, its coordination number, its oxidation state, and the magnetic order of the host material [38]. Figure 12 shows the $U_{\mathrm{SC}-\mathrm{SD}}$ obtained for all inequivalent Mn Hubbard sites in SMO with $\mathrm{Fe}_{\mathrm{Mn}}-\mathrm{V}_{\mathrm{O}}^{\mathrm{OP}}$ defect pairs, where the substitutional $\mathrm{Fe}$ is in $\mathrm{NN}$ (top panels), NNN (middle panels), or NNNN (bottom panels) position relative to $\mathrm{V}_{\mathrm{O}}^{\mathrm{OP}}$. Hubbard parameters are reported for each site as a function of the distance of the $\mathrm{Mn}$ site from the $\mathrm{Fe}_{\mathrm{Mn}}$ (via the color of the data points) and $\mathrm{V}_{\mathrm{O}}^{\mathrm{OP}}$ defects.

\section{a. AFM SMO}

Results for AFM SMO suggest that the distance from the $\mathrm{V}_{\mathrm{O}}$ has the strongest impact on the computed $U$ values [cf. Fig. 12(a)]. In fact, we observe deviations from the self-consistent $U$ computed for the stoichiometric cell $\left(U_{\mathrm{SC}}\right)$, mainly for $\mathrm{Mn}$ atoms adjacent to $\mathrm{V}_{\mathrm{O}}$ at a distance of about $1.90 \AA$, while Mn sites at larger distances recover $U_{\mathrm{SC}}$ (the deviations are as small as $0.02 \mathrm{eV}$ ). For NNN and NNNN configurations, the two Mn sites adjacent to the vacancy show different behaviors. The $\mathrm{Mn}$ atom farthest from the $\mathrm{Fe}_{\mathrm{Mn}}$ defect shows $U$ values slightly higher than $U_{\mathrm{SC}}(+0.08 \mathrm{eV})$ with a behavior similar to that of the Mn sites adjacent to a doubly charged $\mathrm{V}_{O}^{x}$ in $\mathrm{SMO}$, which can be explained in terms of a change in coordination number [38]. Instead, a larger reduction $(-0.41 \mathrm{eV})$ is observed for the $\mathrm{Mn}$ atom closest to the substitutional iron and for the only remaining Mn site adjacent to the $\mathrm{V}_{\mathrm{O}}$ in the case of a $\mathrm{NN} \mathrm{Fe}_{\mathrm{Mn}}-\mathrm{V}_{\mathrm{O}}^{\mathrm{OP}}$ defect couple. This latter behavior was observed for the Mn atoms adjacent to a neutral $\mathrm{V}_{\mathrm{O}}^{\bullet \bullet}$ in SMO due to both the change in coordination number and the change in oxidation state of these reduced $\left(\mathrm{Mn}^{3+}\right)$ sites [38]. Similar conclusions can be drawn for all defect pairs (schematically shown in Fig. 13), including $\mathrm{Fe}_{\mathrm{Mn}}-\mathrm{V}_{\mathrm{O}}^{\mathrm{IP}}$ defect pairs as shown in Fig. 14.

We determine the oxidation state according to the method of Ref. [44], which is based on the occupation matrix of the $d$ orbitals of each Hubbard atom that is available when performing DFT $+U$ calculations. Each $d$ orbital is considered to be fully occupied if the corresponding matrix element is closer to unity than a given threshold. In the AFM phase, we use a threshold of 0.9 for the occupation. These oxidation states confirm the above picture (see Fig. 15), where only one of the 
(a) AFM

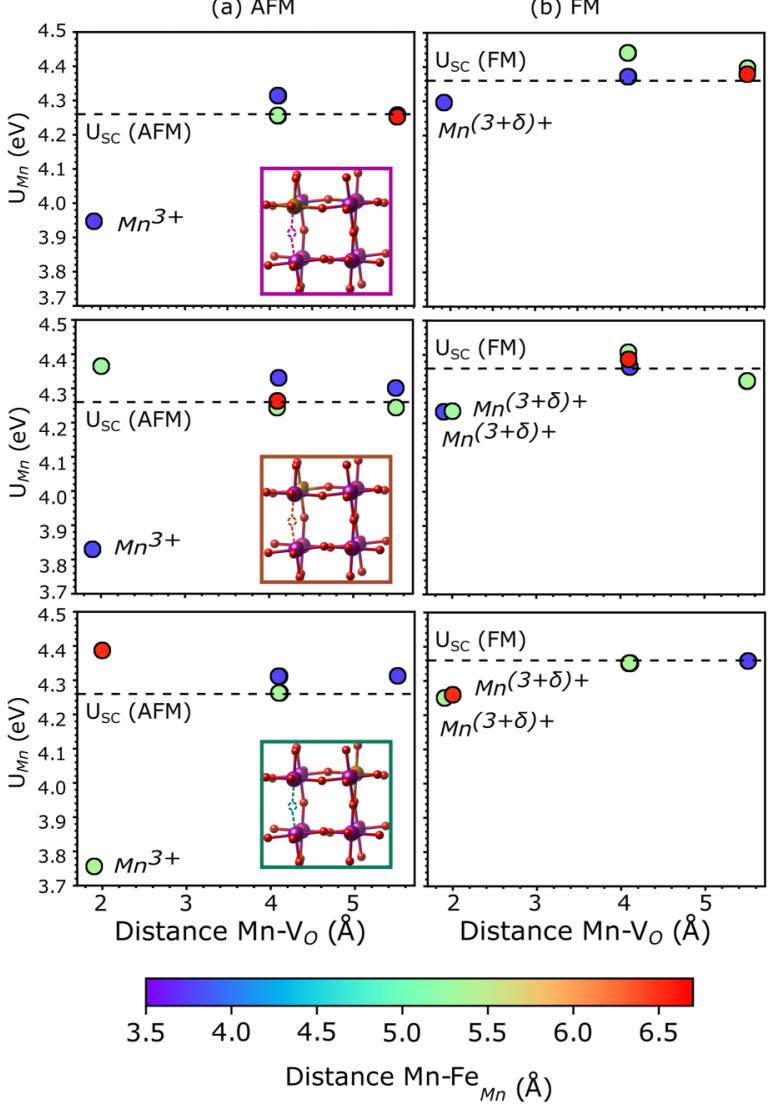

FIG. 12. $U_{\mathrm{SC}-S D}$ values reported as a function of the distance of each Hubbard site from the $\mathrm{V}_{\mathrm{O}}$ and the $\mathrm{Fe}_{\mathrm{Mn}}$ defects, computed for all $\mathrm{Mn}$ atoms in SMO with a NN (top), NNN (middle), and NNNN (bottom) $\mathrm{Fe}_{\mathrm{Mn}}-\mathrm{V}_{\mathrm{O}}^{\mathrm{OP}}$ defect pair in the (a) AFM and (b) FM phases. The oxidation state is explicitly indicated for (partially) reduced $\mathrm{Mn}$ sites. The insets show the structure of the corresponding configuration, where the red, violet, gold, and dashed spheres represent the $\mathrm{O}, \mathrm{Mn}$, and $\mathrm{Fe}$ atoms and the $\mathrm{V}_{\mathrm{O}}$, respectively. The values of $U_{\mathrm{SC}}$ for the stoichiometric phases are indicated by horizontal dashed lines for comparison.

(a) $V_{0}(O P)$

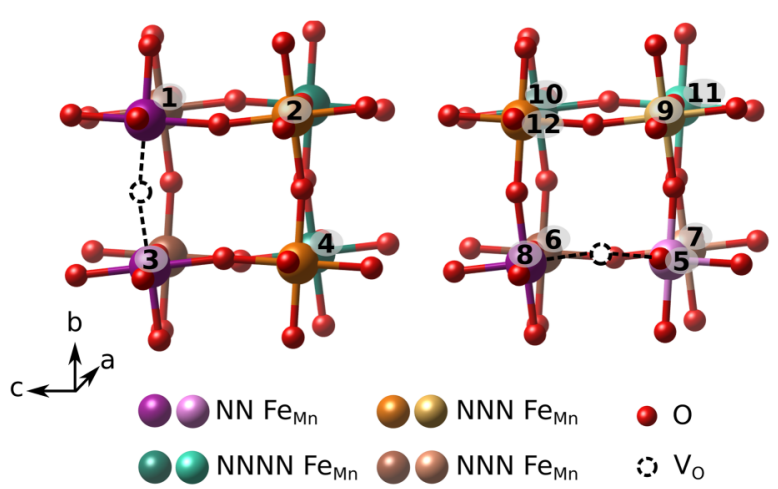

FIG. 13. (a) and (b) Defect-pair configurations as in the main text, numbered according to the location of the Fe substitution site.
Mn sites in nearest-neighbor position relative to the oxygen vacancy is reduced to $\mathrm{Mn}^{3+}$, in line with results of Mössbauer spectroscopy suggesting a partial reduction of the $\mathrm{Mn}$ atoms in $\mathrm{SrMn}_{1-x} \mathrm{Fe}_{x} \mathrm{O}_{3-\delta}$ samples, presumably in the vicinity of the vacant anion [22]. The $\mathrm{Fe}$ ion is always in the $\mathrm{Fe}^{3+}$ charge state, independent of its position relative to the $\mathrm{V}_{\mathrm{O}}$.

\section{b. FM SMO}

For the FM phase [cf. Figs. 12(b) and 16], the $U_{\text {SC-SD }}$ values deviate less from $U_{\mathrm{SC}}$ than for AFM SMO. Interestingly, all the $\mathrm{Mn}$ adjacent to the $\mathrm{V}_{\mathrm{O}}$ show the same behavior with a small reduction of their $U_{\mathrm{SC}-\mathrm{SD}}$ value with respect to $U_{\mathrm{SC}}$, suggesting a partial reduction of these sites $\left(\mathrm{Mn}^{(3+\delta)+}\right)$. These observations can be explained considering the metallic nature of FM SMO, in which one expects the defect state and the corresponding changes in the local chemical environment to be more delocalized over the whole structure, compared with the semiconducting AFM phase, where a more confined impact of the defect on the local chemical environment was already observed in the case of oxygen-deficient SMO [38].

These observations of a partial reduction of multiple Mn sites accompanying the $\mathrm{Fe}^{3+}$ formation are again supported by the determined oxidation states according to the method of Ref. [44]. As opposed to the AFM phase, we use a lower threshold of about 0.7 for the FM order, to be able to discern one (for configurations in which the $\mathrm{V}_{\mathrm{O}}$ is adjacent to one $\mathrm{Mn}$ and to the $\mathrm{Fe}$ ion) or two (in all the other cases) reduced $\mathrm{Mn}$ atoms as shown in Fig. 17.

\section{Hubbard correction for $\mathrm{Fe}: \boldsymbol{U}_{\mathrm{Fe}}$}

We also determined the $U$ value on the Fe using the SC-SD approach as shown in Fig. 18 for each configuration as a function of the $\mathrm{Fe}_{\mathrm{Mn}}-\mathrm{V}_{\mathrm{O}}$ distance. The horizontal dashed lines in Fig. 18 indicate the $U_{\mathrm{SC}}$ value computed for iron in $\mathrm{LaFeO}_{3}$, a perovskite material where $\mathrm{Fe}$ has the same octahedral coordination environment and the same oxidation state $\left(\mathrm{Fe}^{3+}\right)$ as in Fe-doped SMO. Unsurprisingly, larger deviations from these $U_{\mathrm{SC}}$ values are generally observed for configurations (in violet in Fig. 18) in which the $\mathrm{Fe}_{\mathrm{Mn}}$ is in nearest-neighbor position relative to the $\mathrm{V}_{\mathrm{O}}$ and in the insulating AFM phase. The larger deviation of the $U$ value for the $\mathrm{Fe}$ in the NNNN $\mathrm{Fe}_{\mathrm{Mn}}-\mathrm{V}_{\mathrm{O}}^{\mathrm{IP}}$ observed only in the AFM case can be explained considering the interaction of the $\mathrm{Fe}$ atoms in these configurations with the $\mathrm{V}_{\mathrm{O}}$ in the neighboring cell, which is weaker in the more screened FM phase.

\section{APPENDIX B: STRAIN-DEPENDENT DEFECT-PAIR FORMATION ENERGY IN FM SMO}

As shown in Fig. 19 for the FM phase, under compressive strain, the formation energy of $\mathrm{Fe}_{\mathrm{Mn}}-\mathrm{V}_{\mathrm{O}}^{\mathrm{IP}}$ defects increases as expected from volume arguments, which can be explained in terms of a reduced sensitivity of the metallic FM phase to crystal field effects, allowing volume effects to dominate [48].

\section{APPENDIX C: MAGNETIC ORDER IN DFT $+U_{\text {SC }}$}

Figure 20 shows that for DFT $+U_{\mathrm{SC}}$, in which the global $U_{\mathrm{SC}}$ computed for stoichiometric bulk SMO in the corre- 

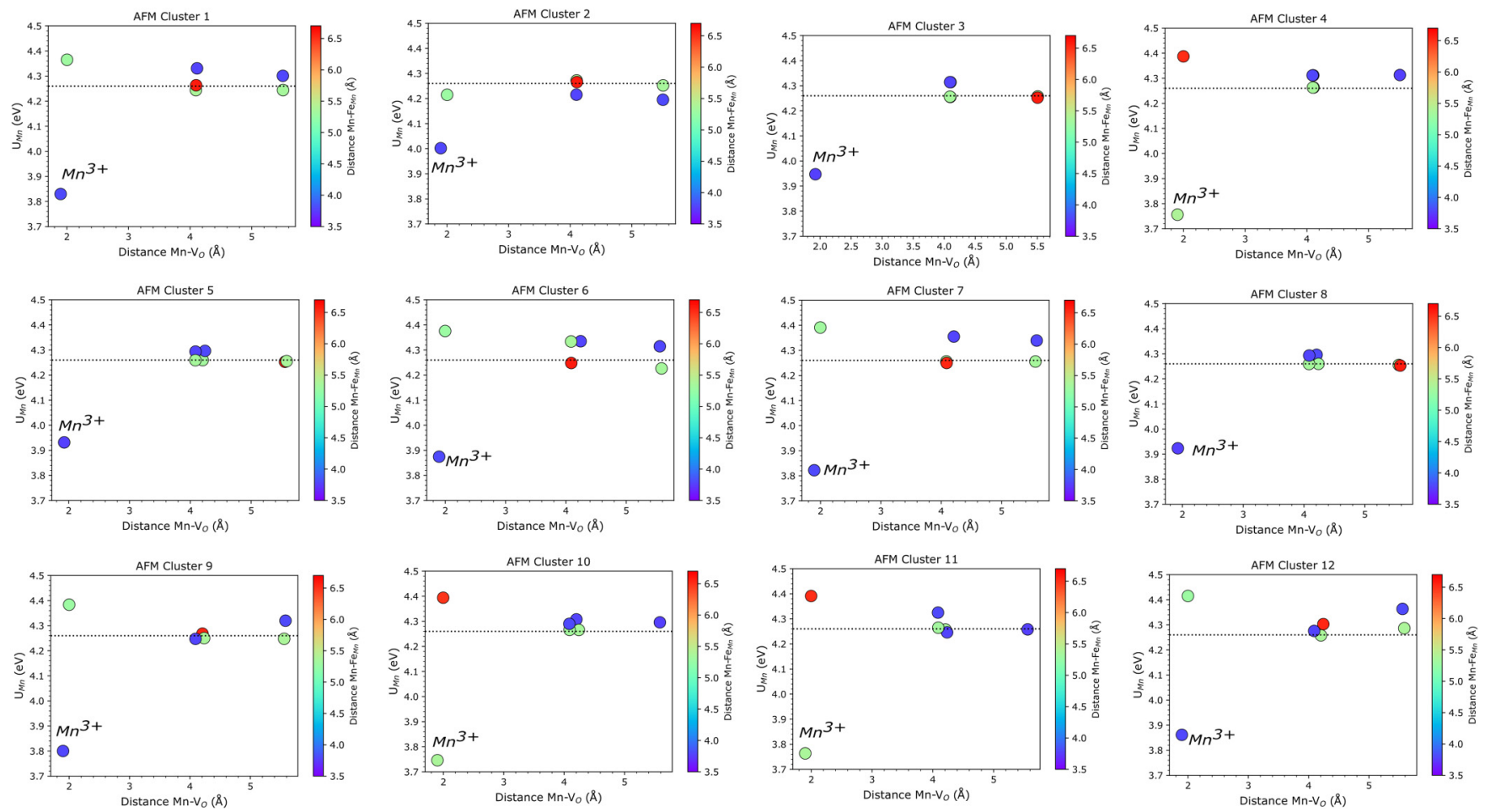

FIG. 14. $U_{\text {SC-SD }}$ values reported as a function of the distance of each Hubbard site from $\mathrm{Fe}_{\mathrm{Mn}}$ and $\mathrm{V}_{\mathrm{O}}$ defects computed for the Mn atoms in AFM SMO structures in each of the $12 \mathrm{Fe}_{\mathrm{Mn}}-\mathrm{V}_{\mathrm{O}}$ configurations shown in Fig. 13. The oxidation state is indicated for the reduced Mn sites.

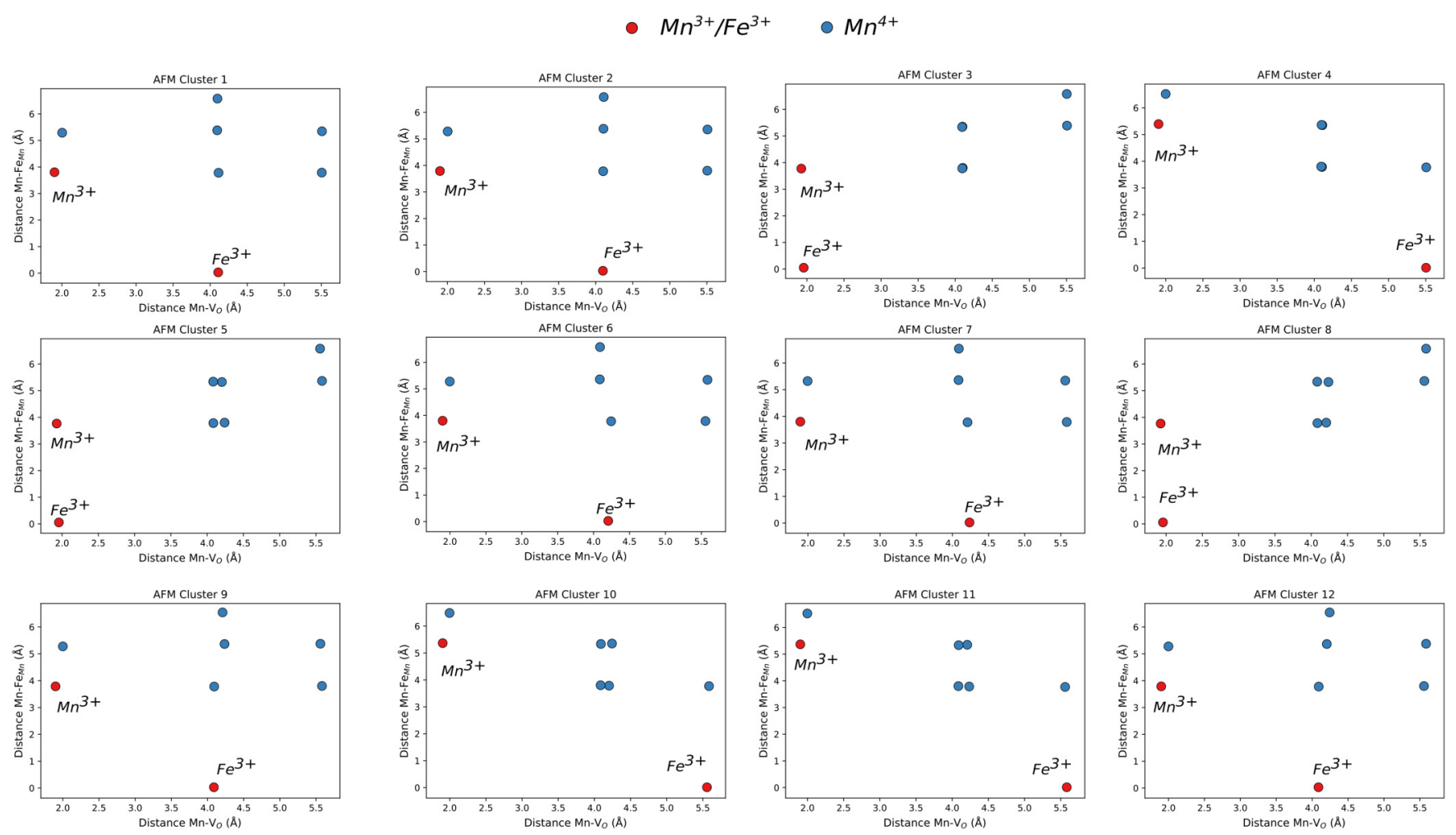

FIG. 15. Oxidation state reported as a function of the distance of each site from $\mathrm{Fe}_{\mathrm{Mn}}$ and $\mathrm{V}_{\mathrm{O}}$ defects computed for the Mn or Fe atoms in AFM SMO structures in each of the $12 \mathrm{Fe}_{\mathrm{Mn}}-\mathrm{V}_{\mathrm{O}}$ configurations shown in Fig. 13. 

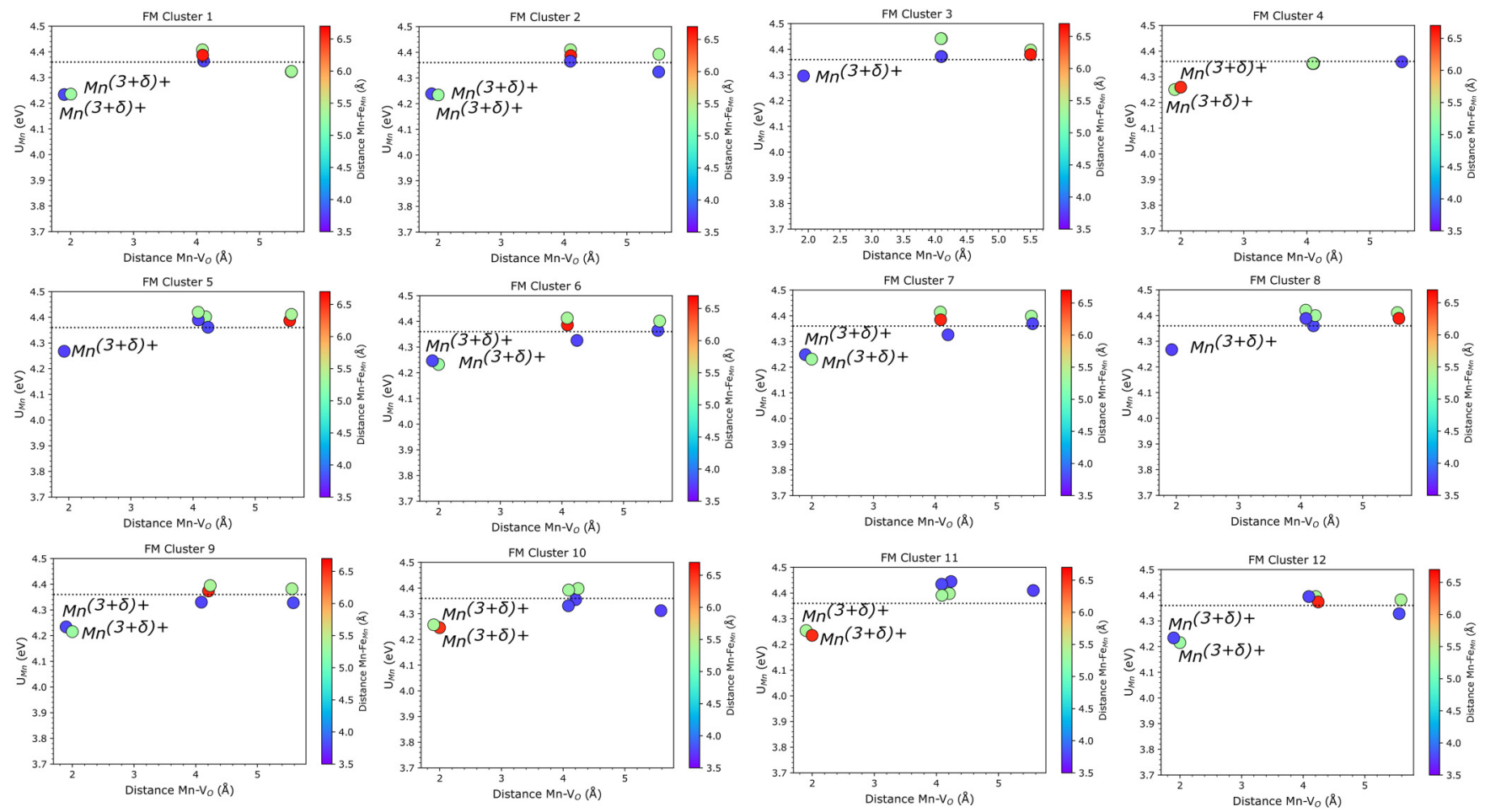

FIG. 16. $U_{\mathrm{SC}-\mathrm{SD}}$ values reported as a function of the distance of each Hubbard site from $\mathrm{Fe}_{\mathrm{Mn}}$ and $\mathrm{V}_{\mathrm{O}}$ defects computed for the Mn atoms in FM SMO structures in each of the $12 \mathrm{Fe}_{\mathrm{Mn}}-\mathrm{V}_{\mathrm{O}}$ configurations shown in Fig. 13. The oxidation state is indicated for the partially reduced Mn sites.
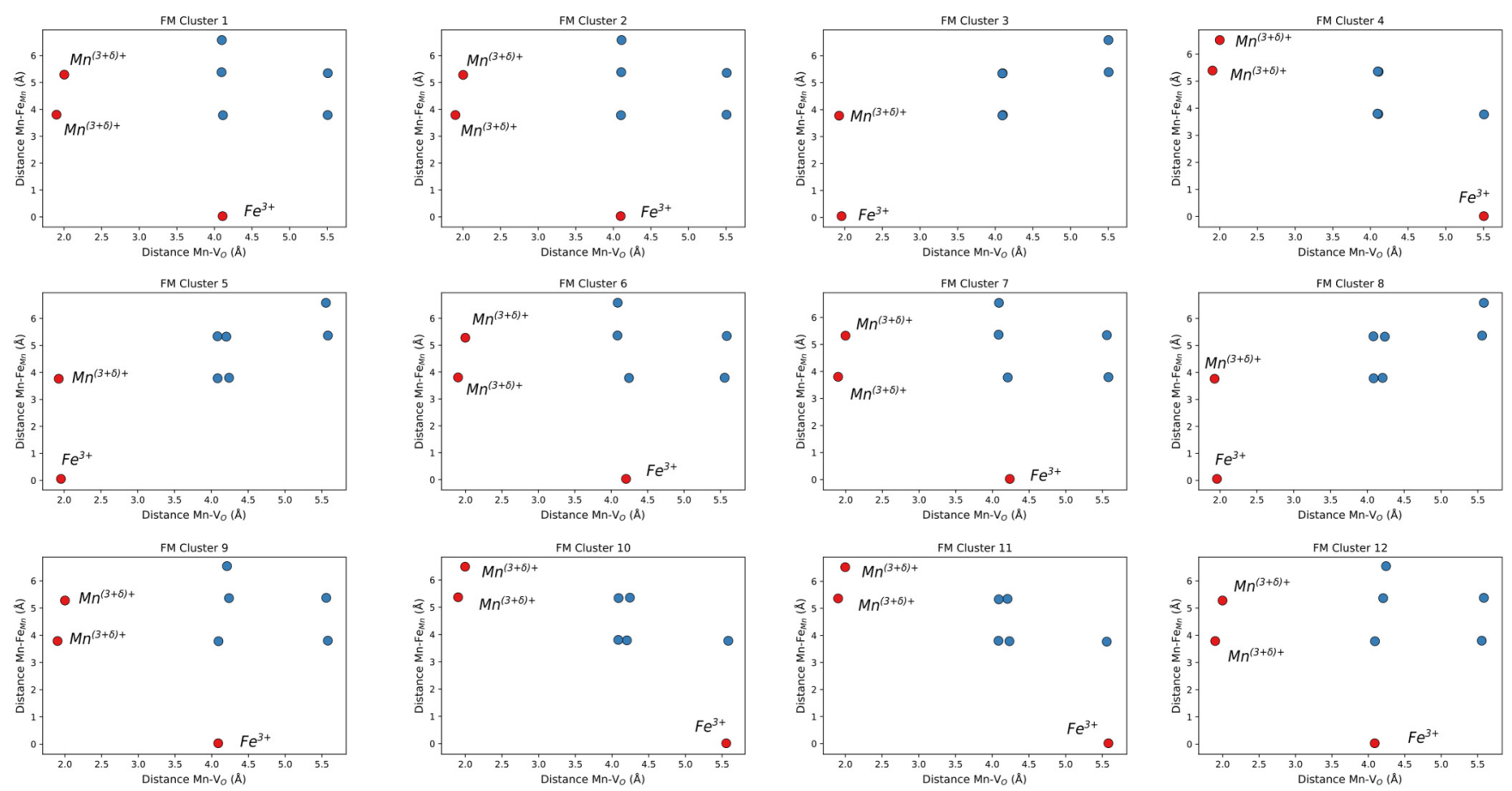

FIG. 17. Oxidation state reported as a function of the distance of each site from $\mathrm{Fe}_{\mathrm{Mn}}$ and $\mathrm{V}_{\mathrm{O}}$ defects computed for the Mn or Fe atoms in FM SMO structures in each of the $12 \mathrm{Fe}_{\mathrm{Mn}}-\mathrm{V}_{\mathrm{O}}$ configurations shown in Fig. 13. 

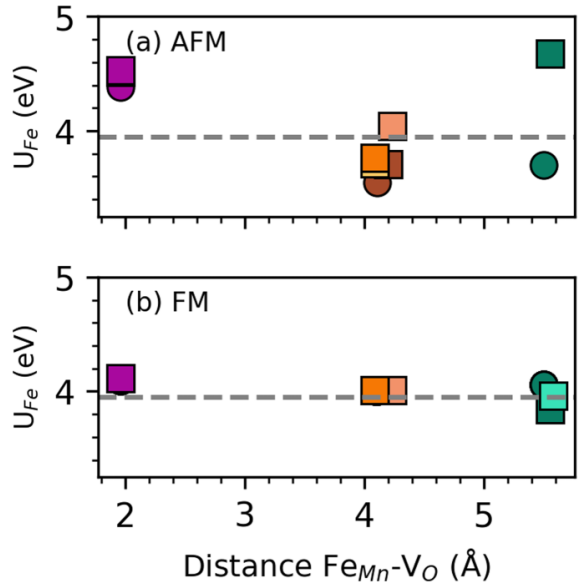

FIG. 18. $U_{\mathrm{SC}-\mathrm{SD}}$ values on the $\mathrm{Fe}_{\mathrm{Mn}}$ atoms computed for all the considered $\mathrm{Fe}_{\mathrm{Mn}}-\mathrm{V}_{\mathrm{O}}$ configurations in (a) AFM and (b) FM SMO. Circles and squares refer to data obtained for $\mathrm{V}_{\mathrm{O}}^{\mathrm{OP}}$ and $\mathrm{V}_{\mathrm{O}}^{\mathrm{IP}}$, respectively. See Fig. 2 of the main text for the color code. The horizontal dashed line indicates the $U_{\mathrm{SC}}$ value computed for $\mathrm{Fe}$ ions in the (a) G-AFM and (b) FM phases of $\mathrm{LaFeO}_{3}$ as a reference.

sponding magnetic phase is applied on all the Mn sites, all the considered $\mathrm{Fe}_{\mathrm{Mn}}-\mathrm{V}_{\mathrm{O}}$ configurations are more stable in the FM phase and that the stability of the FM order increases with increasing distance of $\mathrm{Fe}_{\mathrm{Mn}}$ from $\mathrm{V}_{\mathrm{O}}$. Indeed, the increasing distance between the $\mathrm{Fe}^{3+}$ and the reduced $\mathrm{Mn}^{3+}$ ions promotes the ferromagnetic $\mathrm{Mn}^{3+}$ or $\mathrm{Mn}^{4+}$ interactions.

\section{APPENDIX D: DEFECT-INDUCED POLARIZATION}

\section{Polarization in the unstrained AFM phase}

Figure 21(a) shows that the angle between the polarization $\vec{P}$ and the $\mathrm{Fe}_{\mathrm{Mn}}^{\prime}-\mathrm{V}_{\mathrm{O}}^{\bullet \bullet}$ dipole $\vec{D}$ decreases with increasing the
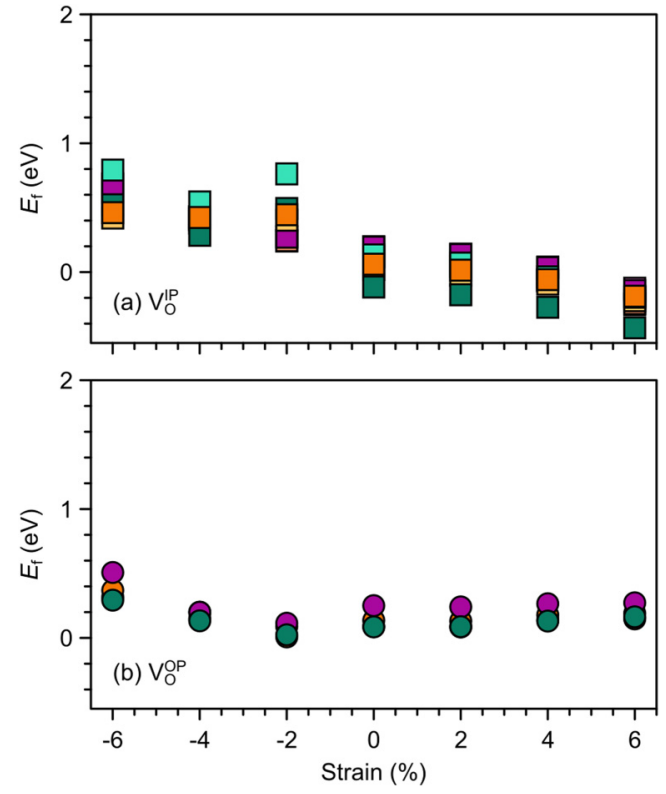

FIG. 19. Strain-dependent formation energy $E_{\mathrm{f}}$ of (a) $\mathrm{Fe}_{\mathrm{Mn}}-\mathrm{V}_{\mathrm{O}}^{\mathrm{IP}}$ and (b) $\mathrm{Fe}_{\mathrm{Mn}}-\mathrm{V}_{\mathrm{O}}^{\mathrm{OP}}$ defect pairs in FM SMO. See color code in Fig. 2 of the main text.

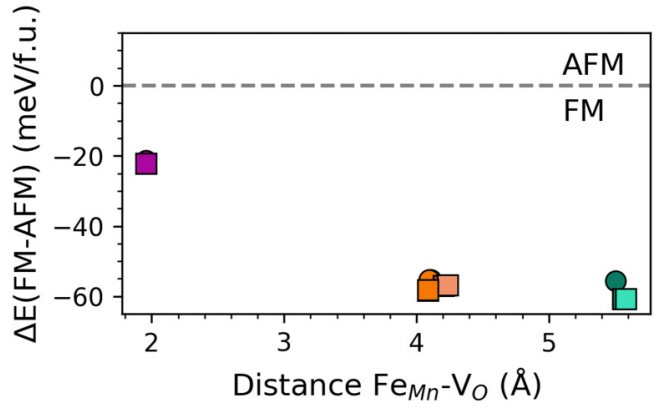

FIG. 20. Total energy differences $[\Delta E$ (FM-AFM)] per formula unit between the defective cells with FM and AFM order computed with the DFT $+U_{\mathrm{SC}}$ approach. $\mathrm{AFM}$ is more stable for positive differences, and FM is more stable for negative differences. $\Delta E(\mathrm{FM}-\mathrm{AFM})$ is reported with respect to the $\mathrm{Fe}_{\mathrm{Mn}}-\mathrm{V}_{\mathrm{O}}$ distance in each defective configuration in the unstrained SMO structure. Circles and squares refer to data obtained for $\mathrm{VO}_{\mathrm{O}}^{\mathrm{OP}}$ and $\mathrm{VO}_{\mathrm{O}}^{\mathrm{IP}}$, respectively. See color code in Fig. 2 of the main text.

$\mathrm{Fe}_{\mathrm{Mn}}^{\prime}-\mathrm{V}_{\mathrm{O}}^{\bullet \bullet}$ distance. This observation seems counterintuitive but can be explained considering also the presence of the $\mathrm{Mn}_{\mathrm{Mn}}^{\prime}-\mathrm{V}_{\mathrm{O}}^{\bullet \bullet}$ dipole $\vec{D}^{\prime}$, with the reduced $\mathrm{Mn}$ always being adjacent to $\mathrm{V}_{\mathrm{O}}$. The closer the $\mathrm{Fe}_{\mathrm{Mn}}^{\prime}, \mathrm{Mn}_{\mathrm{Mn}}^{\prime}$, and $\mathrm{V}_{\mathrm{O}}$ sites, the stronger the coupling between $\vec{D}$ and $\vec{D}^{\prime}$ and consequently the larger the deviation of $\vec{P}$ from $\vec{D}$. Indeed, the polarization is mainly aligned along the direction of the vector sum of the $\vec{D}$ and $\vec{D}^{\prime}$ dipoles $\left(\vec{D}_{\text {tot }}\right)$ as shown by the smaller angles between $\vec{D}_{\text {tot }}$ and $\vec{P}$ [see Fig. $21(\mathrm{~b})$ ].
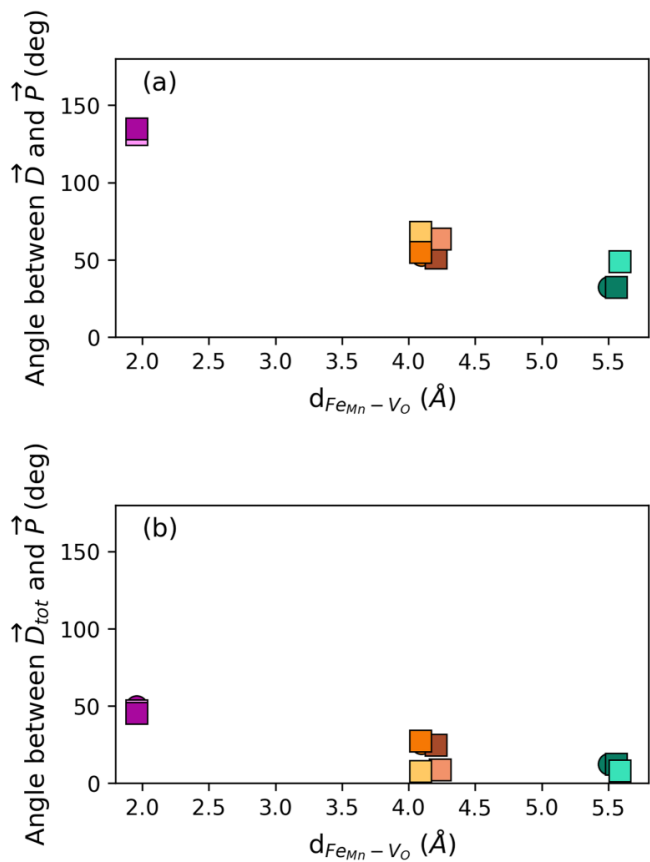

FIG. 21. Angle between (a) the $\mathrm{Fe}_{\mathrm{Mn}}^{\prime}-\mathrm{V}_{\mathrm{O}}^{\bullet \bullet}$ defect dipole $\vec{D}$ or (b) the total defect dipole $\vec{D}_{\text {tot }}$ and the polarization $\vec{P}$ as a function of the distance between $\mathrm{Fe}_{\mathrm{Mn}}$ and $\mathrm{V}_{\mathrm{O}}$ for the different configurations in the AFM phase. Circles and squares refer to data obtained for $\mathrm{V}_{\mathrm{O}}^{\mathrm{OP}}$ and $\mathrm{V}_{\mathrm{O}}^{\mathrm{IP}}$, respectively. See Fig. 2 in the main text for the color code. 

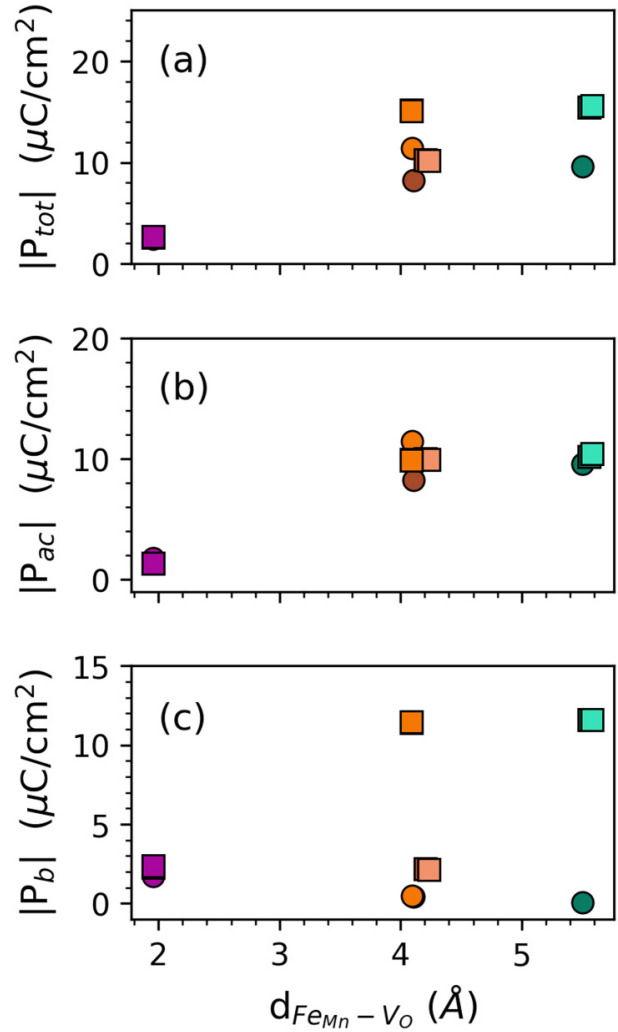

FIG. 22. Modulus of (a) the total polarization vector and (b) the in-plane $\left(\left|\vec{P}_{a c}\right|\right)$ and (c) the out-of-plane $\left(\left|\vec{P}_{b}\right|\right)$ components for the different defect configurations in the FM phase. Circles and squares refer to data obtained for $\mathrm{V}_{\mathrm{O}}^{\mathrm{OP}}$ and $\mathrm{V}_{\mathrm{O}}^{\mathrm{IP}}$, respectively. See Fig. 2 in the main text for the color code.

\section{Polarization in the unstrained FM phase}

The polarization for the FM phase was computed using the nominal charges of +2 for $\mathrm{Sr},-2$ for $\mathrm{O},+3$ for $\mathrm{Fe}$, and +4 or +3 for stoichiometriclike or reduced Mn sites, even though the results of Appendix A $1 \mathrm{~b}$ indicated that, due to the metallic nature of this phase, the reduction of the $\mathrm{Mn}$ sites is only partial. As shown in Fig. 22 while NN defect configurations, with only one reduced $\mathrm{Mn}$ site adjacent to the $\mathrm{V}_{\mathrm{O}}$, exhibit similar or slightly higher total polarization $\vec{P}_{\text {tot }}$ compared with the AFM phase, the NNN and NNNN $\mathrm{Fe}_{\mathrm{Mn}}-\mathrm{V}_{\mathrm{O}}$ defect pairs show almost the same $\vec{P}_{\text {tot }}$, regardless of the $\mathrm{Fe}_{\mathrm{Mn}}-\mathrm{V}_{\mathrm{O}}$ distance, contrarily to the increase in $\vec{P}_{\mathrm{tot}}$ with increasing $\mathrm{Fe}_{\mathrm{Mn}}-\mathrm{V}_{\mathrm{O}}$ distance reported for the AFM phase (cf. Fig. 7 in the main text). This behavior can be explained considering that for these configurations the polarization is computed assuming the presence of two $\mathrm{Mn}_{\mathrm{Mn}}^{\prime}-\mathrm{V}_{\mathrm{O}}^{\bullet \bullet}$ defect pairs involving the $\mathrm{Mn}$ sites adjacent to $\mathrm{V}_{\mathrm{O}}$, which results in the reduction or cancellation of the contribution to the polarization along the axis of the broken $\mathrm{Mn}-\mathrm{O}-\mathrm{Mn}$ bonds. For example, for all the $\mathrm{Fe}_{\mathrm{Mn}}-\mathrm{V}_{\mathrm{O}}^{\mathrm{OP}}$ configurations the out-of-plane $\vec{P}_{b}$ is almost null, the broken Mn-O-Mn bond lying along the $b$ axis. The observed behavior for the FM phase can thus be explained considering the presence of only one defect dipole (the one associated with $\mathrm{Fe}_{\mathrm{Mn}}^{\prime}-\mathrm{V}_{\mathrm{O}}^{\bullet \bullet}$ ) inducing the polarization, with respect to the AFM phase where both $\mathrm{Fe}_{\mathrm{Mn}}^{\prime}-\mathrm{V}_{\mathrm{O}}^{\bullet \bullet}$ and $\mathrm{Mn}_{\mathrm{Mn}}^{\prime}-\mathrm{V}_{\mathrm{O}}^{\bullet \bullet}$ are responsible for the predicted properties.
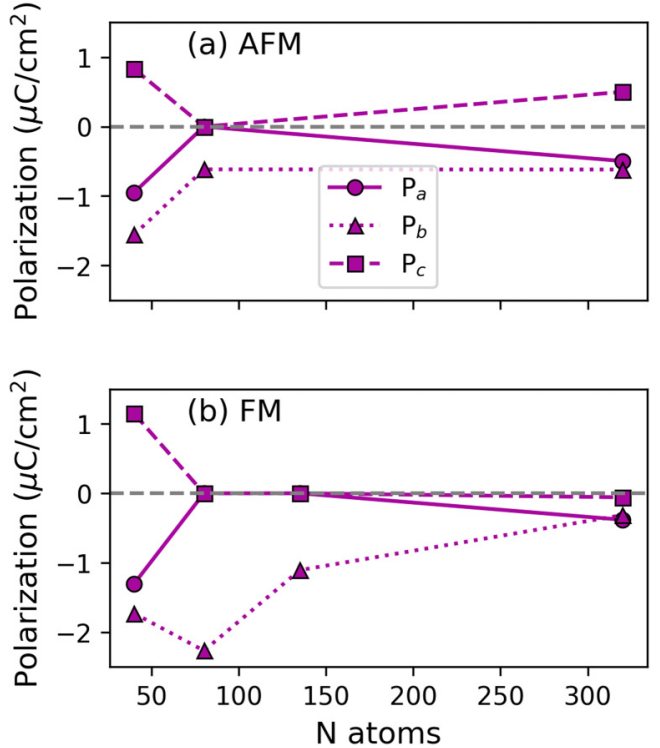

FIG. 23. Evolution of the $a, b$, and $c$ components of the polarization for the $\mathrm{NN} \mathrm{Fe}_{\mathrm{Mn}}-\mathrm{V}_{\mathrm{O}}^{\mathrm{OP}}$ defective supercell as a function of the cell size in (a) the AFM and (b) the FM phase.

\section{Polarization as a function of the cell size}

As shown in Fig. 23, we generally observe a reduction of the polarization with increasing cell size, deviations such as those in the 80-atom cell likely being associated with cell-anisotropy effects. This suggests a local polarizing effect that becomes less important as the cell size increases and hence the defect-pair concentration decreases.

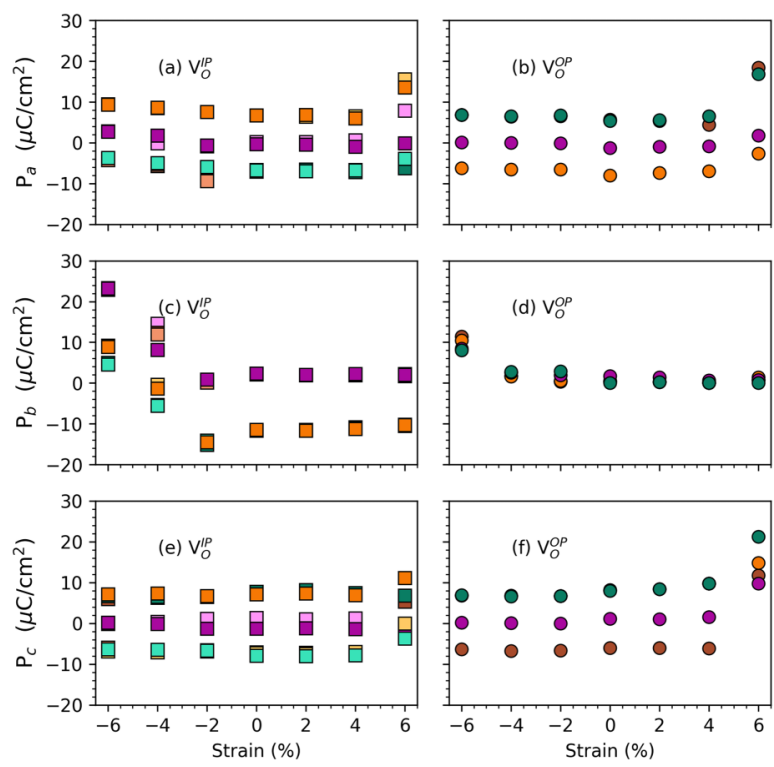

FIG. 24. Epitaxial-strain-driven changes of the (a) and (b) $a$, (c) and (d) $b$, and (e) and (f) $c$ components of the polarization for the different defect-pair configurations in the FM phase of SMO. Plots are for (a), (c), and (e) $\mathrm{Fe}_{\mathrm{Mn}}-\mathrm{V}_{\mathrm{O}}^{\mathrm{OP}}$ and (b), (d), and (f) $\mathrm{Fe}_{\mathrm{Mn}}-\mathrm{V}_{\mathrm{O}}^{\mathrm{IP}}$ defects. Circles and squares refer to data obtained for $\mathrm{V}_{\mathrm{O}}^{\mathrm{OP}}$ and $\mathrm{V}_{\mathrm{O}}^{\mathrm{IP}}$, respectively. See Fig. 2 in the main text for the color code. 

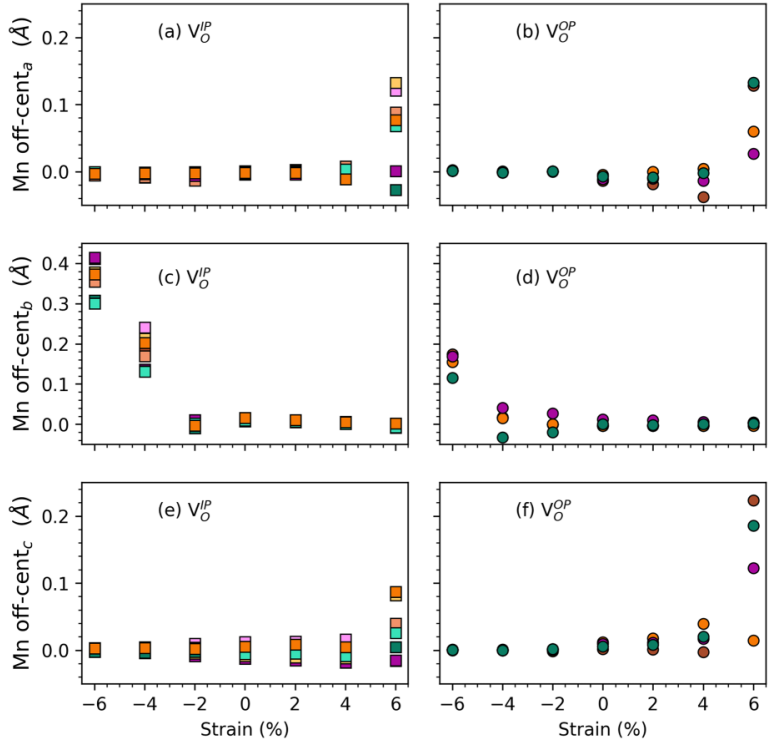

FIG. 25. Strain dependence of the average Mn off-centering along the (a) and (b) $a$ axis, (c) and (d) $b$ axis, and (e) and (f) $c$ axis for the different defect-pair configurations in the FM phase of SMO. Plots are for (a), (c), and (e) $\mathrm{Fe}_{\mathrm{Mn}}-\mathrm{V}_{\mathrm{O}}^{\mathrm{OP}}$ and (b), (d), and (f) $\mathrm{Fe}_{\mathrm{Mn}}-\mathrm{V}_{\mathrm{O}}^{\mathrm{IP}}$ defects. Circles and squares refer to data obtained for $\mathrm{V}_{\mathrm{O}}^{\mathrm{OP}}$ and $\mathrm{V}_{\mathrm{O}}^{\mathrm{IP}}$, respectively. See Fig. 2 in the main text for the color code.

\section{Interplay between strain and polarization in the FM phase}

The strain-dependent polarization of Fe-doped oxygendeficient SMO is the result of a complex interplay between defect chemistry and electronic and magnetic degrees of freedom. While for the insulator AFM phase, polarization can be enhanced by strain, in the FM phase the polarization is fairly constant with respect to the applied epitaxial strain (see Fig. 24), and the average Mn off-centerings remain very small (about $0.02-0.04 \AA$ in the strain range between -2 and $4 \%$; see Fig. 25), similar in magnitude to the unstrained structure. Interestingly, compressive strain values of $4 \%$ can result in an enhancement of the $P_{b}$ component, as confirmed also by the larger Mn off-centerings observed at this strain along the $b$ axis. Also at $6 \%$ tensile strain an increase in the components of the polarization and of the Mn off-centerings in the $a c$ plane is observed. This behavior can be understood considering the larger electronic screening of the defect dipole in the metallic FM phase, resulting in the lower sensitivity of the polarization of the defective cells to the applied strain and evolution with strain of the frequency of the polar modes in the stoichiometric FM order, where the IP modes soften only for large tensile strain of $6 \%$ and the OP modes becomes slightly unstable at $2 \%$ compressive strain [17].
[1] K. Fuchigami, Z. Gai, T. Z. Ward, L. F. Yin, P. C. Snijders, E. W. Plummer, and J. Shen, Tunable Metallicity of the $\mathrm{La}_{5 / 8} \mathrm{Ca}_{3 / 8} \mathrm{MnO}_{3}(001)$ Surface by an Oxygen Overlayer, Phys. Rev. Lett. 102, 066104 (2009).

[2] H. L. Tuller and S. R. Bishop, Point defects in oxides: Tailoring materials through defect engineering, Annu. Rev. Mater. Res. 41, 369 (2011).

[3] S. V. Kalinin, A. Borisevich, and D. Fong, Beyond condensed matter physics on the nanoscale: The role of ionic and electrochemical phenomena in the physical functionalities of oxide materials, ACS Nano 6, 10423 (2012).

[4] S. V. Kalinin and N. A. Spaldin, Functional ion defects in transition metal oxides, Science 341, 858 (2013).

[5] A. Chandrasekaran, D. Damjanovic, N. Setter, and N. Marzari, Defect ordering and defect-domain-wall interactions in $\mathrm{PbTiO}_{3}$ : A first-principles study, Phys. Rev. B 88, 214116 (2013).

[6] N. Biškup, J. Salafranca, V. Mehta, M. P. Oxley, Y. Suzuki, S. J. Pennycook, S. T. Pantelides, and M. Varela, Insulating Ferromagnetic $\mathrm{LaCoO}_{3-\delta}$ Films: A Phase Induced by Ordering of Oxygen Vacancies, Phys. Rev. Lett. 112, 087202 (2014).

[7] A. Bhattacharya and S. J. May, Magnetic oxide heterostructures, Annu. Rev. Mater. Res. 44, 65 (2014).

[8] C. Becher, L. Maurel, U. Aschauer, M. Lilienblum, C. Magén, D. Meier, E. Langenberg, M. Trassin, J. Blasco, I. P. Krug, P. A. Algarabel, N. A. Spaldin, J. A. Pardo, and M. Fiebig, Strain-induced coupling of electrical polarization and structural defects in $\mathrm{SrMnO}_{3}$ films, Nat. Nanotechnol. 10, 661 (2015).

[9] A. Marthinsen, C. Faber, U. Aschauer, N. A. Spaldin, and S. M. Selbach, Coupling and competition between ferroelectricity, magnetism, strain, and oxygen vacancies in $\mathrm{AMnO}_{3}$ perovskites, MRS Commun. 6, 182 (2016).
[10] S. M. Griffin, M. Reidulff, S. M. Selbach, and N. A. Spaldin, Defect chemistry as a crystal structure design parameter: Intrinsic point defects and Ga substitution in $\mathrm{InMnO}_{3}, \mathrm{Chem}$. Mater. 29, 2425 (2017).

[11] T. Rojac and D. Damjanovic, Domain walls and defects in ferroelectric materials, Jpn. J. Appl. Phys. 56, 10PA01 (2017).

[12] J. H. Lee and K. M. Rabe, Epitaxial-Strain-Induced Multiferroicity in $\mathrm{SrMnO}_{3}$ from First Principles, Phys. Rev. Lett. 104, 207204 (2010).

[13] Y.-G. Wang, X.-G. Tang, Q.-X. Liu, Y.-P. Jiang, and L.-L. Jiang, Room temperature tunable multiferroic properties in sol-gel-derived nanocrystalline $\operatorname{Sr}\left(\mathrm{Ti}_{1-x} \mathrm{Fe}_{x}\right) \mathrm{O}_{3-\delta}$ thin films, Nanomaterials 7, 264 (2017).

[14] Y. Syono, Y. Akimoto, and K. Kohn, Structure relations of hexagonal perovskite-like compounds $\mathrm{ABX}_{3}$ at high pressure, J. Phys. Soc. Jpn. 26, 993 (1969).

[15] O. Chmaissem, B. Dabrowski, S. Kolesnik, J. Mais, D. E. Brown, R. Kruk, P. Prior, B. Pyles, and J. D. Jorgensen, Relationship between structural parameters and the Néel temperature in $\mathrm{Sr}_{1-\mathrm{x}} \mathrm{Ca}_{\mathrm{x}} \mathrm{MnO}_{3}(0 \leqslant \mathrm{x} \leqslant 1)$ and $\mathrm{Sr}_{1-\mathrm{y}} \mathrm{Ba}_{\mathrm{y}} \mathrm{MnO}_{3}(\mathrm{y} \leqslant$ 0.2), Phys. Rev. B 64, 134412 (2001).

[16] S. Kobayashi, Y. Tokuda, T. Ohnishi, T. Mizoguchi, N. Shibata, Y. Sato, Y. Ikuhara, and T. Yamamoto, Cation off-stoichiometric $\mathrm{SrMnO}_{3-\delta}$ thin film grown by pulsed laser deposition, J. Mater. Sci. 46, 4354 (2010).

[17] C. Ricca, D. Berkowitz, and U. Aschauer, Ferroelectricity promoted by cation/anion divacancies in $\mathrm{SrMnO}_{3}$, arXiv:2105.09360 [J. Mater. Chem. C (to be publsihed)].

[18] I. D. Fawcett, G. M. Veith, M. Greenblatt, M. Croft, and I. Nowik, Properties of the perovskites, $\mathrm{SrMn}_{1-x} \mathrm{Fe}_{x} \mathrm{O}_{3-\delta}(x=$ 1/3, 1/2, 2/3), Solid State Sci. 2, 821 (2000). 
[19] S. Jin, T. H. Tiefel, M. McCormack, R. A. Fastnacht, R. Ramesh, and L. H. Chen, Thousandfold change in resistivity in magnetoresistive La-Ca-Mn-O films, Science 264, 413 (1994).

[20] A. J. Millis, Lattice effects in magnetoresistive manganese perovskites, Nature (London) 392, 147 (1998).

[21] P. D. Battle, T. C. Gibb, and C. W. Jones, The structural and magnetic properties of $\mathrm{SrMnO}_{3}$ : A reinvestigation, J. Solid State Chem. 74, 60 (1988).

[22] P. D. Battle, C. M. Davison, T. C. Gibb, and J. F. Vente, Structural chemistry of $\mathrm{SrMn}_{1-x} \mathrm{Fe}_{x} \mathrm{O}_{3-\delta}, x \approx 0.3$, J. Mater. Chem. 6, 1187 (1996).

[23] P. Giannozzi, S. Baroni, N. Bonini, M. Calandra, R. Car, C. Cavazzoni, D. Ceresoli, G. L. Chiarotti, M. Cococcioni, I. Dabo, A. Dal Corso, S. de Gironcoli, S. Fabris, G. Fratesi, R. Gebauer, U. Gerstmann, C. Gougoussis, A. Kokalj, M. Lazzeri, L. Martin-Samos et al., QUANTUM ESPRESSO: A modular and open-source software project for quantum simulations of materials, J. Phys.: Condens. Matter 21, 395502 (2009).

[24] P. Giannozzi, O. Andreussi, T. Brumme, O. Bunau, M. Buongiorno Nardelli, M. Calandra, R. Car, C. Cavazzoni, D. Ceresoli, M. Cococcioni, N. Colonna, I. Carnimeo, A. Dal Corso, S. De Gironcoli, P. Delugas, R. A. Distasio, A. Ferretti, A. Floris, G. Fratesi, G. Fugallo et al., Advanced capabilities for materials modelling with QUANTUM ESPRESSO, J. Phys.: Condens. Matter 29, 465901 (2017).

[25] J. P. Perdew, A. Ruzsinszky, G. I. Csonka, O. A. Vydrov, G. E. Scuseria, L. A. Constantin, X. Zhou, and K. Burke, Restoring the Density-Gradient Expansion for Exchange in Solids and Surfaces, Phys. Rev. Lett. 100, 136406 (2008).

[26] D. Vanderbilt, Soft self-consistent pseudopotentials in a generalized eigenvalue formalism, Phys. Rev. B 41, 7892 (1990).

[27] Ultrasoft pseudopotentials from the PSLIBRARY were taken from https://www.materialscloud.org: Sr.pbesol-spnrrkjus_psl.1.0.0.UPF, Mn.pbesol-spn-rrkjus_ps1.0.3.1.UPF, and O.pbesol-n-rrkjus_psl.1.0.0.UPF.

[28] H. J. Monkhorst and J. D. Pack, Special points for Brillouinzone integrations, Phys. Rev. B 13, 5188 (1976).

[29] J. M. Rondinelli and N. A. Spaldin, Structure and properties of functional oxide thin films: Insights from electronic-structure calculations, Adv. Mater. (Weinheim) 23, 3363 (2011).

[30] F. A. Kröger and H. J. Vink, Relations between the concentrations of imperfections in crystalline solids, Solid State Phys. 3, 307 (1956).

[31] P. Graziosi, A. Gambardella, M. Prezioso, A. Riminucci, I. Bergenti, N. Homonnay, G. Schmidt, D. Pullini, and D. Busquets-Mataix, Polaron framework to account for transport properties in metallic epitaxial manganite films, Phys. Rev. B 89, 214411 (2014).

[32] P. Erhart, A. Klein, D. Åberg, and B. Sadigh, Efficacy of the $\mathrm{DFT}+U$ formalism for modeling hole polarons in perovskite oxides, Phys. Rev. B 90, 035204 (2014).

[33] G. Geneste, B. Amadon, M. Torrent, and G. Dezanneau, DFT + $U$ study of self-trapping, trapping, and mobility of oxygen-type hole polarons in barium stannate, Phys. Rev. B 96, 134123 (2017).

[34] G. Geneste, C. Paillard, and B. Dkhil, Polarons, vacancies, vacancy associations, and defect states in multiferroic $\mathrm{BiFeO}_{3}$, Phys. Rev. B 99, 024104 (2019).
[35] V. I. Anisimov, J. Zaanen, and O. K. Andersen, Band theory and Mott insulators: Hubbard $U$ instead of Stoner $I$, Phys. Rev. B 44, 943 (1991).

[36] V. I. Anisimov, A. I. Poteryaev, M. A. Korotin, A. O. Anokhin, and G. Kotliar, First-principles calculations of the electronic structure and spectra of strongly correlated systems: Dynamical mean-field theory, J. Phys.: Condens. Matter 9, 7359 (1997).

[37] S. L. Dudarev, G. A. Botton, S. Y. Savrasov, C. J. Humphreys, and A. P. Sutton, Electron-energy-loss spectra and the structural stability of nickel oxide: An LSDA+U study, Phys. Rev. B 57, 1505 (1998).

[38] C. Ricca, I. Timrov, M. Cococcioni, N. Marzari, and U. Aschauer, Self-consistent site-dependent DFT $+U$ study of stoichiometric and defective $\mathrm{SrMnO}_{3}$, Phys. Rev. B 99, 094102 (2019).

[39] I. Timrov, N. Marzari, and M. Cococcioni, Hubbard parameters from density-functional perturbation theory, Phys. Rev. B 98 085127 (2018).

[40] C. Freysoldt, B. Grabowski, T. Hickel, J. Neugebauer, G. Kresse, A. Janotti, and C. G. Van de Walle, First-principles calculations for point defects in solids, Rev. Mod. Phys. 86, 253 (2014).

[41] S. Lany and A. Zunger, Assessment of correction methods for the band-gap problem and for finite-size effects in supercell defect calculations: Case studies for $\mathrm{ZnO}$ and GaAs, Phys. Rev. B 78, 235104 (2008).

[42] C. G. Van de Walle and J. Neugebauer, First-principles calculations for defects and impurities: Applications to III-nitrides, J. Appl. Phys. (Melville, NY) 95, 3851 (2004).

[43] A. Jain, G. Hautier, S. P. Ong, C. J. Moore, C. C. Fischer, K. A. Persson, and G. Ceder, Formation enthalpies by mixing GGA and GGA $+U$ calculations, Phys. Rev. B 84, 045115 (2011).

[44] P. H. L. Sit, R. Car, M. H. Cohen, and A. Selloni, Simple, unambiguous theoretical approach to oxidation state determination via first-principles calculations, Inorg. Chem. 50, 10259 (2011).

[45] R. D. King-Smith and D. Vanderbilt, Theory of polarization of crystalline solids, Phys. Rev. B 47, 1651 (1993).

[46] D. Vanderbilt and R. D. King-Smith, Electric polarization as a bulk quantity and its relation to surface charge, Phys. Rev. B 48 , 4442 (1993).

[47] https://doi.org/10.24435/materialscloud:d0-fb.

[48] U. Aschauer, R. Pfenninger, S. M. Selbach, T. Grande, and N. A. Spaldin, Strain-controlled oxygen vacancy formation and ordering in $\mathrm{CaMnO}_{3}$, Phys. Rev. B 88, 054111 (2013).

[49] U. Aschauer and N. A. Spaldin, Interplay between strain, defect charge state, and functionality in complex oxides, Appl. Phys. Lett. 109, 031901 (2016).

[50] S. B. Adler, Factors governing oxygen reduction in solid oxide fuel cell cathodes, Chem. Rev. (Washington, DC) 104, 4791 (2004).

[51] C. Ederer and N. A. Spaldin, Effect of Epitaxial Strain on the Spontaneous Polarization of Thin Film Ferroelectrics, Phys. Rev. Lett. 95, 257601 (2005).

[52] C. Varvenne, F. Bruneval, M.-C. Marinica, and E. Clouet, Point defect modeling in materials: Coupling $a b$ initio and elasticity approaches, Phys. Rev. B 88, 134102 (2013).

[53] D. Khomskii, Classifying multiferroics: Mechanism and effect, Physics 2, 20 (2009).

[54] http://www.id.unibe.ch/hpc. 